\title{
Empirical Assessment of Bifurcation Regions within New Keynesian Models
}

\author{
by \\ William A. Barnett, University of Kansas* \\ and \\ Evgeniya Aleksandrovna Duzhak, Baruch College, City University of New York**
}

October 23, 2008

\begin{abstract}
As is well known in systems theory, the parameter space of most dynamic models is stratified into subsets, each of which supports a different kind of dynamic solution. Since we do not know the parameters with certainty, knowledge of the location of the bifurcation boundaries is of fundamental importance. Without knowledge of the location of such boundaries, there is no way to know whether the confidence region about the parameters' point estimates might be crossed by one or more such boundaries. If there are intersections between bifurcation boundaries and a confidence region, the resulting stratification of the confidence region damages inference robustness about dynamics, when such dynamical inferences are produced by the usual simulations at the point estimates only.

Recently, interest in policy in some circles has moved to New Keynesian models, which have become common in monetary policy formulations. As a result, we explore bifurcations within the class of New Keynesian models. We study different specifications of monetary policy rules within the New Keynesian functional structure. In initial research in this area, Barnett and Duzhak (2008) found a New Keynesian Hopf bifurcation boundary, with the setting of the policy parameters influencing the existence and location of the bifurcation boundary. Hopf bifurcation is the most commonly encountered type of bifurcation boundary found among economic models, since the existence of a Hopf bifurcation boundary is accompanied by regular oscillations within a neighborhood of the bifurcation boundary. Now, following a more extensive and systematic search of the parameter space, we also find the existence of Period Doubling (flip) bifurcation boundaries in the class of models.

Central results in this research are our theorems on the existence and location of Hopf bifurcation boundaries in each of the considered cases. We also solve numerically for the location and properties of the Period Doubling bifurcation boundaries and their dependence upon policy-rule parameter settings.
\end{abstract}

\section{Keywords:}

Bifurcation, dynamic general equilibrium, Hopf bifurcation, flip bifurcation, period doubling bifurcation, robustness, New Keynesian macroeconometrics, Taylor rule, inflation targeting.

JEL Codes:

C14, C22, E37, E32.

\footnotetext{
* Department of Economics, University of Kansas, Lawrence, KS 66045, Phone: 785-864-2844, E-mail: barnett@ku.edu

** Zicklin School of Business, Baruch College, City University of New York, One Bernard Baruch Way, Box B10-225, New York, NY 10010, Phone: (646)-312-3474,E-mail: Evgeniya_duzhak@baruch.cuny.edu
} 


\section{Introduction}

During the past 30 years, the literature in macroeconomics has moved from comparative statics to dynamics, with many such dynamical models exhibiting nonlinear dynamics. The core of dynamics is bifurcation theory, which is fundamental to systems theory. The parameter space is stratified into subsets, each of which supports a different kind of dynamic solution. But dynamic econometric inferences are usually produced from simulations with the parameters set only at the point estimates, rather than at various settings within confidence regions around the point estimates. Without knowledge of the location of bifurcation boundaries, there is no way to know whether the confidence region about the parameters' point estimates might be crossed by such a boundary, thereby stratifying the confidence region itself and damaging inference robustness regarding dynamics.

Bifurcation refers to a change in qualitative features of the solution dynamics as parameter values change. Without bifurcation, we observe a change in quantitative features of dynamic solutions, such as change in period or amplitude of cycles, as parameters change, rather than qualitative features of dynamics, such as change from monotonic convergence to damped convergence to a steady state. A parameter-space point at which change in a quality of the solution occurs defines a point on a bifurcation boundary within the space of parameters. Close to a bifurcation boundary the system quantitative features become more sensitive to changes in the parameters on one side of the bifurcation boundary. Small alternations of parameters can cause large changes in quantitative characteristics of the solution dynamics, but qualitative changes occur only when the parameters cross a bifurcation boundary.

There are different types of bifurcation, such as flip, fold, singularity, transcritical, and Hopf. Each of these bifurcations produces a different type of qualitative change in dynamics. Hopf bifurcation is the most commonly seen type among economic models, since the existence of a Hopf bifurcation boundary is accompanied by regular oscillations in an economic model, when the parameters 
are within a neighborhood of the boundary, and where the oscillations may damp to a stable steady state or may never damp, depending upon the side of the bifurcation boundary on which the parameters might lie. ${ }^{1}$ Clearly the precision of the point estimates of parameters is critical, when the point estimate is near a bifurcation boundary, since different qualitative properties of the solution can exist within the confidence region, if a bifurcation boundary crosses the confidence region.

The first theoretical work on Hopf bifurcation appeared in Poincaré (1892). The first formulation of a theorem on Hopf bifurcation appeared in Andronov (1929), who, with his coauthors, developed important tools for analyzing nonlinear dynamical systems. A famous general theorem on the existence of Hopf bifurcation was proved by Hopf (1942). While the work of Poincaré and Andronov was concerned with two-dimensional vector fields, the theorem of Hopf is valid in $n$ dimensions. When parameters cross a bifurcation boundary such that the solutions change from damped stable to unstable limit cycles, it is common in mathematics to refer to the resulting bifurcation as Poincaré-Andronov-Hopf bifurcation.

Hopf bifurcation boundaries have been found in many economic models, such as Torre (1977) and Benhabib and Nishimura (1979). These were among the first works on Hopf bifurcation in the field of economics. Torre studied Keynesian systems and found the appearance of a limit cycle associated with a Hopf bifurcation boundary. Benhabib and Nishimura analyzed a multi-sector neoclassical optimal growth model and showed that a closed invariant curve might emerge as the result of optimization. Historically, optimal growth theory received the most attention as the subject of bifurcation analysis. Hopf bifurcations were also found in overlapping generations models. ${ }^{2}$ These studies illustrate that the existence of a Hopf bifurcation boundary in an economic model results in a solution following closed curves around the stationary state, with the solution paths being stable or unstable, depending upon which side of the bifurcation boundary contains the parameter values. More recent studies finding bifurcation in econometric models include Barnett and $\mathrm{He}$ (1999, 2001, 2002, 2004, 2006, 2008), finding bifurcation boundaries within the

\footnotetext{
${ }^{1}$ See, e.g., Kuznetsov (1998), and Seydel (1994).

${ }^{2}$ See Aiyagari (1989), Benhabib and Day (1982), Benhabib and Rustichini (1991), Gale (1973).
} 
parameter spaces of the Bergstrom continuous-time model of the UK economy and the Leeper and Sims Euler-equations model of the United States economy.

New Keynesian models have become increasingly popular in policy analysis. The usual New-Keynesian log-linearized model consists of a forward-looking IScurve, describing consumption smoothing behavior, and a New Keynesian Phillips curve, derived from price optimization by monopolistically competitive firms in the presence of nominal rigidities. The third equation is a monetary policy rule. This paper continues and substantially extends our initial more-limited bifurcation analysis of New Keynesian functional structure in Barnett and Duzhak (2008). We use eigenvalues of the linearized system of difference equations to locate Hopf bifurcation boundaries. We also investigate the effects of different monetary policy rules on bifurcation boundary locations. In each case, we solve numerically for the location and properties of the bifurcation boundary and its dependency upon policy rule parameter settings. We use two types of New Keynesian models. One type can be reduced to produce a $2 \times 2$ Jacobian. The other type produces a $3 \times 3$ Jacobian. To our knowledge there is no theoretical literature for the $3 \times 3$ case in the economics literature. In the $3 \times 3$ case we employ the theorem on Hopf bifurcation from the engineering literature.

The rest of the paper is structured as follows. In section 2 we lay out the basic New Keynesian model and monetary policy rules used. Monetary policy rules include different specifications of Taylor's rule and inflation targeting. Section 3 introduces the basic definitions and theorems of bifurcation theory employed in this paper and applies bifurcation theory to New Keynesian models. We consider ten cases, with different monetary policy rules incorporated into the model. We formulate and prove propositions establishing the existence of Hopf bifurcation. Beyond that analytical analysis, we use numerical techniques to identify existence of period doubling bifurcation in some of the cases. Section 4 concludes.

\section{Model}

We base our analysis on the New Keynesian functional structure described in this section. The main assumption of New Keynesian economic theory is that there 
are nominal price rigidities preventing prices from adjusting immediately and thereby creating disequilibrium unemployment. Price stickiness is often introduced in the manner proposed by Calvo (1983). The model below, used as the theoretical background for our log linearized bifurcation analysis, is based closely upon Walsh (2003), section 5.4.1, pp. 232 - 239, which in turn is based upon the monopolistic competition model of Dixit and Stiglitz (1977). ${ }^{3}$

The model consists of consumers, firms, and monetary policy authority. Consumers derive utility from the composite consumption good, $\mathrm{C}_{\mathrm{t}}$, real money balances, and leisure. Consumers supply their labor in a competitive labor market and receive labor income, $W_{t} N_{t}$. Consumers own the firms producing consumption goods and receive all profits, $\Pi_{t}$. The representative consumer can allocate wealth to money and bonds and choose the aggregate consumption stream by solving the utility maximization problem.

Firms operate in a monopolistically competitive market. Each firm has pricing power over the goods it sells. Price rigidity by the firm results from the fact that a random fraction of firms does not adjust its product price in each period. The remaining firms adjust prices to their optimal levels. Firms make their production and price-setting decisions by solving the cost minimization and pricing decision problems, such that

$$
\begin{aligned}
x_{t} & =\mathrm{E}_{\mathrm{t}} x_{t+1}-\left(i_{t}-\mathrm{E}_{\mathrm{t}} \pi_{t+1}\right) / \sigma, \\
\pi_{t} & =\beta E_{t} \pi_{t+1}+\kappa x_{t},
\end{aligned}
$$

where, $\pi_{t}$ is the inflation rate at time $\mathrm{t} ; i_{t}$ is the interest rate; $x_{t}=\left(\hat{y}_{t}-\hat{y}_{t}^{f}\right)$ is the gap between actual output percentage deviation, $\hat{y}_{t}$, and the flexible-price output percentage deviation, $\hat{y}_{t}^{f} ; \sigma$ is a degree of relative risk aversion; $E_{t}$ is the expectations operator conditionally upon information at time $\mathrm{t}$, and $\beta$ is the discount factor.

We now have two equations. The first equation, (2.1), provides the demand side of the economy and is a forward-looking IS curve that relates the output gap to

\footnotetext{
${ }^{3}$ Other relevant references include Shapiro (2006) and Woodford (2003).
} 
the real interest rate. Equation (2.2) is the New-Keynesian Phillips curve, which represents the supply side by describing how inflation is driven by the output gap and expected inflation. The resulting system of two equations has three unknown variables: inflation, output gap, and nominal interest rate.

We need one more equation to close the model. The remaining necessary equation will be a monetary policy rule, in which the central bank uses a nominal interest rate as the policy instrument. Numerous types of monetary policy rules have been discussed in the economics literature. Two main policy classes are targeting rules and instrument rules.

A simple instrument rule relates the interest rate to a few observable variables. The most famous such rule is Taylor's rule. Taylor demonstrated that a simple reaction function, with a short-term interest-rate policy instrument, responding to inflation and output gap, follows closely the observed path of the Federal Funds rate. His original work was followed by a large literature, in which researchers have tried to modify Taylor's rule to acquire a better fit to the data. ${ }^{4}$ We initially center our analysis on the following specification of the current-looking Taylor rule:

$$
i_{t}=a_{1} \pi_{t}+a_{2} x_{t}
$$

where $a_{1}$ is the coefficient of the central bank's reaction to inflation and $a_{2}$ is the coefficient of the central bank's reaction to the output gap. We also consider the forward-looking, backward-looking, and the hybrid Taylor rules.

Among targeting rules, the recent literature proposes many ways to define an inflation target. ${ }^{5}$ We consider inflation targeting policies of the form:

$$
i_{t}=a_{1} \pi_{t}
$$

which is a current-looking inflation targeting rule. Forward-looking and backwardlooking inflation targeting will also be considered.

\footnotetext{
4See, e.g., Clarida, Gali and Gertler (1999); Gali and Gertler (1999); McCallum (1999); and Taylor (1999).

5 See Bernanke et al. (1999), Svensson (1999), and Gavin (2003).
} 
When we use the current-looking Taylor rule, we are left with these three equations:

$$
\begin{aligned}
& x_{t}=E_{t} x_{t+1}-\frac{1}{\sigma}\left(i_{t}-E_{t} \pi_{t+1}\right), \\
& \pi_{t}=\beta E_{t} \pi_{t+1}+\kappa x_{t}, \\
& i_{t}=a_{1} \pi_{t}+a_{2} x_{t} .
\end{aligned}
$$

This 3-equation system constitutes a New Keynesian model.

\subsection{Determinacy and Stability Analysis}

In order to analyze the models' determinacy and stability properties, we need to display it in the standard form:

$$
\mathrm{E}_{\mathrm{t}} \mathrm{x}_{t+1}=\mathrm{Cx}_{\mathrm{t}}+\boldsymbol{\delta}_{t}
$$

where $\boldsymbol{\delta}_{t}$ is a vector of disturbances and is the expectations operator conditional upon information in period $t$. With the current-looking Taylor rule, we need the following version, which is not in closed form:

$$
\begin{aligned}
& \mathbf{A E} \mathbf{x}_{t+1}=\mathrm{Bx}_{\mathrm{t}}+\boldsymbol{\delta}_{t} \text {, where } \\
& \mathbf{A}=\left[\begin{array}{lll}
1 & \frac{1}{\sigma} & 0 \\
0 & \beta & 0 \\
0 & 0 & 0
\end{array}\right], \mathbf{B}=\left[\begin{array}{ccc}
1 & 0 & \frac{1}{\sigma} \\
-\kappa & 1 & 0 \\
a_{2} & a_{1} & -1
\end{array}\right], \mathbf{x}_{\mathrm{t}}=\left[\begin{array}{c}
x_{t} \\
\pi_{t} \\
i_{t}
\end{array}\right] .
\end{aligned}
$$

Obtaining the matrix $\mathbf{C}=\mathrm{A}^{-1} \mathrm{~B}$ in this case is impossible, because $\mathrm{A}$ is a singular matrix.

Therefore, we reduce the system of three equations to a system of two loglinearized equations by substituting Taylor's rule into the consumption Euler equation. The resulting system of expected difference equations has a determinate solution, if the number of eigenvalues outside the unit circle equals the number of forward looking variables (see Blanchard and Kahn (1980)). That system of two equations has the following form:

$$
\left[\begin{array}{cc}
1 & \frac{1}{\sigma} \\
0 & \beta
\end{array}\right]\left[\begin{array}{l}
E_{t} x_{t+1} \\
E_{t} \pi_{t+1}
\end{array}\right]=\left[\begin{array}{cc}
1+\frac{a_{2}}{\sigma} & -\frac{a_{1}}{\sigma} \\
-\kappa & 1
\end{array}\right]\left[\begin{array}{l}
x_{t} \\
\pi_{t}
\end{array}\right],
$$

which can be written as

$$
\mathrm{AE}_{\mathrm{t}} \mathbf{x}_{\mathrm{t}+1}=\mathrm{Bx}_{\mathrm{t}}
$$


where $\mathbf{x}_{t}=\left[\begin{array}{l}x_{t} \\ \pi_{t}\end{array}\right], \mathbf{A}=\left[\begin{array}{ll}1 & \frac{1}{\sigma} \\ 0 & \beta\end{array}\right]$, and $\mathbf{B}=\left[\begin{array}{cc}1+\frac{a_{2}}{\sigma} & -\frac{a_{1}}{\sigma} \\ -\kappa & 1\end{array}\right]$.

Premultiply the system by the inverse matrix $\mathrm{A}^{-1}$,

$$
\mathbf{A}^{-1}=\left[\begin{array}{cc}
1 & -\frac{1}{\beta \sigma} \\
0 & \frac{1}{\beta}
\end{array}\right],
$$

we get

$$
E_{t} \mathbf{x}_{t+1}=\mathbf{C} \mathbf{x}_{t}
$$

or

$$
\left[\begin{array}{l}
E_{t} x_{t+1} \\
E_{t} \pi_{t+1}
\end{array}\right]=\left[\begin{array}{cc}
1+\frac{a_{2} \beta+\kappa}{\sigma \beta} & \frac{a_{1} \beta-1}{\sigma \beta} \\
-\frac{\kappa}{\beta} & \frac{1}{\beta}
\end{array}\right]\left[\begin{array}{l}
x_{t} \\
\pi_{t}
\end{array}\right]
$$

where $\mathbf{C}=\mathbf{A}^{-1} \mathbf{B}$.

We have two forward-looking variables, $x_{t+1}$ and $\pi_{t+1}$. Therefore uniqueness and stability of the solution require both eigenvalues to be outside the unit circle. The eigenvalues of $\mathbf{C}$ are the roots of the characteristic polynomial

$$
\begin{aligned}
& p(\lambda)=\operatorname{det}(\mathbf{C}-\lambda \mathbf{I}) \\
& =\lambda^{2}-\lambda\left[1+\frac{a_{2} \beta+k}{\sigma \beta}+\frac{1}{\beta}\right]+\frac{\sigma \beta+a_{2} \beta+k a_{1} \beta}{\sigma \beta^{2}} .
\end{aligned}
$$

Defining $D$ as

$$
D=\left[1+\frac{a_{2} \beta+k}{\sigma \beta}+\frac{1}{\beta}\right]^{2}-4 \frac{\sigma \beta+a_{2} \beta+k a_{1} \beta}{\sigma \beta^{2}},
$$

we can write the eigenvalues as

$$
\lambda_{1}=\left(\frac{1}{2}\right)\left(1+\frac{a_{2} \beta+\kappa}{\sigma \beta}+\frac{1}{\beta}+\sqrt{D}\right) \text { and } \lambda_{2}=\left(\frac{1}{2}\right)\left(1+\frac{a_{2} \beta+\kappa}{\sigma \beta}+\frac{1}{\beta}-\sqrt{D}\right) .
$$

It can be shown that both eigenvalues will be outside the unit circle, if and only if 


$$
\left(a_{1}-1\right) \kappa+(1-\beta) a_{2}>0 .
$$

Equivalently, (2.9) holds if $a_{1}>1$. Interest rate rules that meet this criterion are called active. This relationship is also known as Taylor's principle, which prescribes that the interest rate should be set higher than the increase in inflation. Monetary policy satisfying the Taylor's principle is thought to eliminate equilibrium multiplicities. Assuming uniqueness of solutions, the dynamical properties of the system can be explored through bifurcation analysis.

\section{Bifurcation Analysis}

The New Keynesian model has both a continuous time and a discrete form. To define our notation for the discrete form, we consider a continuously differentiable map

$$
\mathbf{x} \mapsto \mathbf{f}(\mathbf{x}, \boldsymbol{\varphi}),
$$

Where $\mathrm{x}$ is an $\mathrm{n}$-dimensional vector of variables, $\varphi$ is an $\mathrm{m}$-dimensional parameter vector, and $\mathrm{f}$ is continuously differentiable, mapping into $\mathrm{n}$-dimensional Euclidean space. We will study the dynamic solution behavior of $\mathbf{x}$ as $\varphi$ varies. System (3.1) undergoes a bifurcation, if its parameters pass through a critical (bifurcation) point, defined as follows.

Definition 3.1: Appearance of a topologically nonequivalent phase portrait under variation of parameters is called a bifurcation.

In other words, bifurcation occurs once a free parameter $\varphi_{\mathrm{i}}$ crosses through a critical bifurcation value, and the quality features of the solution for map (3.1) change. Varying a parameter, thus, results in a transition from a quantitative change to a qualitative change. At the bifurcation point the structure may change its stability, split into new structures, merge with other structures, or produce even more complex behavior. After conducting bifurcation analysis, one can split a parameter set into several subsets. Each of these subsets represents a different type of dynamics. 
There are two possible bifurcation analyses: local and global. We linearize and look at small neighborhoods of a fixed point, $x^{*}=f\left(x^{*}, \varphi\right)$, to conduct local bifurcation analysis. In the case of global analysis, bifurcation boundaries are located without linearization.

Definition 3.2: A local bifurcation is a bifurcation that is identified conditionally upon linearization around a single invariant set or attractor.

The local bifurcations of a map (3.1) can be characterized by the eigenvalues of the Jacobian of the first derivatives of the map, computed at the bifurcation point. Let $\mathbf{J}=\mathbf{f}(\mathbf{x}, \varphi)_{\mathbf{x}}$ be the Jacobian matrix with respect to $\mathbf{x}$. The eigenvalues, $\lambda_{1}, \lambda_{2}, \ldots, \lambda_{n}$, of the Jacobian are also referred to as multipliers. Bifurcation will occur, if there are eigenvalues of $\mathrm{J}$ on the unit circle that violate the following hyperbolicity condition.

Definition 3.3: The equilibrium is called hyperbolic, when the Jacobian J has no eigenvalues on the unit circle.

Non-hyperbolic equilibria usually are not structurally stable and often lead to bifurcations as a parameter is varied. There are three possible ways to violate the hyperbolicity condition. They give rise to three codimension-1 types of bifurcations.

Definition 3.4: Bifurcation associated with the appearance of $\lambda_{i}=1$ is called a fold (or tangent) bifurcation.

Definition 3.5: Bifurcation associated with the appearance of $\lambda_{i}=-1$ is called flip (period-doubling) bifurcation. 
Definition 3.6: Bifurcation corresponding to the presence of a pair of complex conjugate eigenvalues, $\lambda_{1}=e^{+i \theta_{0}}$ and $\lambda_{2}=e^{-i \theta_{0}}$, for $0<\theta_{0}<\pi$, is called a Hopf bifurcation.

However, it is not always the case that nonhyperbolicity induces the appearance of bifurcations. One needs to look at derivatives conditions in addition to hyperbolicity, as we shall see in the theorems below.

In the 2-dimensional case, we shall need the following theorem, based upon the version of the Hopf Bifurcation Theorem presented in Gandolfo (1996, ch. 25, p. 492).

Theorem 3.1: (Existence of Hopf Bifurcation in 2 Dimensions) Consider a map $\mathbf{x} \mapsto \mathbf{f}(\mathbf{x}, \boldsymbol{\varphi})$, where $\mathbf{x}$ has 2 dimensions. For each $\boldsymbol{\varphi}$ in the relevant region, assume that there is a continuously differentiable family of equilibrium points, $\mathbf{x}^{*}=\mathbf{x}^{*}(\varphi)$, at which the eigenvalues of the Jacobian are complex conjugates, $\lambda_{1}=\theta(\mathbf{x}, \varphi)+i \omega(\mathbf{x}, \varphi)$ and $\lambda_{2}=\theta(\mathbf{x}, \varphi)-i \omega(\mathbf{x}, \varphi)$. Suppose that for one of those equilibria, $\left(\mathbf{x}^{*}, \varphi^{*}\right)$, there is a critical value $\varphi_{i}^{c}$ for one of the parameters, $\varphi_{i}^{*}$, in $\varphi^{*}$ such that:

(a) The modulus of the eigenvalues becomes unity at $\varphi=\varphi^{*}$, but the eigenvalues are not roots of unity. Formally, $\lambda_{1}, \lambda_{2} \neq 1$ and $\bmod \left(\lambda_{1}\right)=\bmod \left(\lambda_{2}\right)=$ $\sqrt{\theta^{2}+\omega^{2}}=1$.

Also suppose that

(b) $\left.\frac{\partial\left|\lambda_{j}\left(\mathbf{x}^{*}, \boldsymbol{\varphi}^{*}\right)\right|}{\partial \varphi_{i}^{*}}\right|_{\varphi_{i}^{*}=\varphi_{i}^{c}} \neq 0 \quad$ for $\mathrm{j}=1,2$.

Then there is an invariant closed curve Hopf-bifurcating from $\varphi^{*}{ }^{6}$

Condition (b) implies that the eigenvalue crosses imaginary axes with nonzero speed as $\varphi$ changes. This theorem applies only to the $2 \times 2$ Jacobian case. We use it for the analysis of the reduced $2 \times 2$ model, $\mathrm{AE}_{\mathrm{t}} \mathbf{X}_{\mathrm{t}+1}=\mathbf{B} \mathbf{x}_{\mathrm{t}}$. The more general

\footnotetext{
${ }^{6}$ Note that we use the notations $\bmod \left(\lambda_{j}\right)$ and $\left|\lambda_{j}\right|$ interchangeably to designate modulus of a complex variable.
} 
case requires the rest of the eigenvalues to have a real part less than zero. In the three-equation case with current-looking or backward-looking policy rules, it can be shown that the only form of bifurcation that is possible with the linearized model is Hopf bifurcation. The broader range of bifurcation types possible with the nonlinear model will be the subject of future research.

The $3 \times 3$ case requires different tools. While analyzing a system with a $3 \times$ 3 dimensional Jacobian, we employ the following Theorem 3.2. Although this theorem is employed in the engineering literature, we have not seen this theorem used in the economics literature. If the dynamic system satisfies Theorem 3.2 and the eigenvalue-crossing condition is satisfied, then there is a closed invariant curve Hopf bifurcation from $\left(\mathrm{x}^{*}, \varphi^{*}\right)$. In proving the theorem, we'll need the following lemma from Wen et al (2002).

Lemma 3.1: For a matrix, $\mathbf{A}=\left(a_{i j}\right)$, with $i, j=1,2,3$, a pair of complex conjugate eigenvalues lies on the unit circle and another eigenvalue lies inside the unit circle, if and only if

(a) $|x|<1$

(b) $|z+x|<1+y$,

(c) $y-x z=1-x^{2}$,

where $z, y$, and $x$ are the coefficients of the characteristic equation $\lambda^{3}+z \lambda^{2}+y \lambda+x=0$ of the matrix, $\mathrm{A}$

The first condition of this lemma ensures that at least one of the eigenvalues will be inside the unit circle. The third condition states that not all of the roots lie 
inside of the unit circle. According to the second condition there shouldn't be any real roots located on the unit circle.

Theorem 3.2: (Existence of Hopf Bifurcation in 3 Dimensions) Consider a map $\mathbf{x} \mapsto \mathbf{f}(\mathbf{x}, \boldsymbol{\varphi})$, where $\mathbf{x}$ has 3 dimensions. Let J be the Jacobian of the transformation, and let the characteristic polynomial of the Jacobian be $\mathrm{P}(\lambda)=$ $\lambda^{3}+z \lambda^{2}+y \lambda+x=0$. Assume that for one of the equilibria, $\left(\mathbf{x}^{*}, \varphi^{*}\right)$, there is a critical value $\varphi_{i}^{c}$ for one of the parameters, $\varphi_{i}^{*}$, in $\varphi^{*}$ such that eigenvalue conditions (a), (b), and (c) and transversality condition (d) hold, where :
(a) $|x|<1$,
(b) $|z+x|<1+y$,
(c) $y-x z=1-x^{2}$,
(d) $\left.\frac{\partial\left|\lambda_{j}\left(\mathbf{x}^{*}, \varphi^{*}\right)\right|}{\partial \varphi_{i}^{*}}\right|_{\varphi_{i}^{*}=\varphi_{i}^{c}} \neq 0 \quad$ for the complex conjugates with $\mathrm{j}=1,2$.

Then there is an invariant closed curve Hopf-bifurcating from $\boldsymbol{\varphi}^{*}$.

Proof: Since $\mathbf{x}$ has dimension 3, Lemma 3.1 applies. Hence if conditions (a), (b), and (c) hold, the Jacobian matrix J has a pair of complex conjugate eigenvalues having modulus equal to 1 and another eigenvalue inside the unit circle. That follows from Lemma 3.1.

If the tranversality condition (d) also holds, then according to the classical Hopf bifurcation criterion in Iooss (1979, p. 33), there exists a Hopf bifurcation.

\subsection{Current-Looking Taylor Rule}

The Jacobian of the New Keynesian model can be written in the form:

$$
\mathbf{J}=\left[\begin{array}{cc}
1+\frac{a_{2} \beta+\kappa}{\sigma \beta} & \frac{a_{1} \beta-1}{\sigma \beta} \\
-\frac{\kappa}{\beta} & \frac{1}{\beta}
\end{array}\right] .
$$


We apply the Hopf bifurcation existence theorem (3.1) to the Jacobian of the loglinearized New Keynesian model, $\mathrm{AE}_{\mathrm{t}} \mathbf{x}_{\mathrm{t}+1}=\mathbf{B} \mathbf{x}_{\mathrm{t}}$.

We need to choose a bifurcation parameter to vary, while holding other parameters constant. The model is parameterized by:

$$
\boldsymbol{\varphi}=\left(\begin{array}{c}
\beta \\
\sigma \\
\kappa \\
a_{1} \\
a_{2}
\end{array}\right) .
$$

Candidates for a bifurcation parameter are coefficients for the monetary policy rule, $a_{1}$ and $a_{2}$. For a New Keynesian model with current looking Taylor rule, we use the following proposition, which we proved in Barnett and Duzhak (2008).

Proposition 3.1: The new Keynesian model with current-looking Taylor rule, (2.1), (2.2), and (2.3), undergoes a Hopf bifurcation, if and only if the discriminant of the characteristic equation is negative and $a_{2}^{c}=\sigma \beta-\kappa a_{1}-\sigma$.

We can combine the critical value for $a_{2}$ with the condition on the discriminant of the characteristic polynomial to provide the condition defining the Hopf bifurcation boundary. The bifurcation boundary is the set of parameter values satisfying the following condition:

$$
-1<\frac{\sigma+\sigma \beta-\kappa a_{1} \beta+\kappa}{\sigma \beta^{2}}<1 .
$$

\subsection{Forward-Looking Taylor Rule}

A forward-looking Taylor rule sets the interest rate according to expected future inflation rate and output gap, in accordance with the following equation:

$$
i_{t}=a_{1} E_{t} \pi_{t+1}+a_{2} E_{t} x_{t+1} .
$$

We need to choose a bifurcation parameter to vary, while holding other parameters constant. The model is parameterized by: 


$$
\boldsymbol{\varphi}=\left(\begin{array}{c}
\beta \\
\sigma \\
\kappa \\
a_{1} \\
a_{2}
\end{array}\right) .
$$

Candidates for a bifurcation parameter are coefficients, $a_{1}$ and $a_{2}$, for the monetary policy rule. We use the following proposition, proved in Barnett and Duzhak (2008).

Proposition 3.2: The New Keynesian model with forward-looking Taylor Rule, (2.1), (2.2), and (3.2), undergoes a Hopf bifurcation, if and only if the discriminant of the characteristic equation is negative and $a_{2}^{c}=-\frac{\sigma}{\beta}+\sigma$.

We can combine the critical value for $a_{2}$ with the condition on the discriminant of the characteristic polynomial to provide the condition defining the Hopf bifurcation boundary. The bifurcation boundary is the set of parameter values satisfying the following condition:

$$
-1<\frac{1}{2}\left(\beta+\frac{\kappa\left(1-a_{1}\right)}{\sigma}+\frac{1}{\beta}\right)<1 .
$$

We numerically analyze the forward-looking Taylor rule for the possibility of period doubling bifurcation. The algorithm for numerical bifurcation analysis is based on the following technique. Given the $\mathrm{i}^{\text {th }}$ iterate of the fixed point, $f^{i}(\mathbf{x})-\mathbf{x}=0$, a period doubling bifurcation is detected according to the following test function:

$$
\varphi_{P D}=\operatorname{det}\left(\mathbf{J}^{(i)}+\mathbf{I}_{n}\right),
$$

where $\mathbf{J}^{(i)}$ is the Jacobian matrix of the iterated map $f^{i}$. A period-doubling bifurcation will occur whenever $\varphi_{P D}=0$. 
Given the standard calibration (see Appendix, Table 1), we locate the bifurcation boundary, displayed in Figure 1. Location of the period-doubling bifurcation boundary is implemented using the software continuation package CONTENT. This dynamical system software is developed by Yuri Kuznetsov and V.V. Levitin.

To locate a bifurcation boundary, we need to choose an initial parameter to vary. We select parameter $a_{2}$ to be a free parameter for numerical bifurcation analysis of the new Keynesian model with forward-looking Taylor's rule. We find a period-doubling bifurcation point at $a_{2}=2.994$ with the other parameters set as in the appendix table. We begin with that setting, in numerically locating the bifurcation boundary as we also vary $a_{1}$. In Figure 1, we display a period-doubling bifurcation boundary that we located as a function of two control parameters: $a_{1}$ and $a_{2}$, with the other parameters set in accordance with Table 1 in the appendix.

The nature of the state space solution will depend upon the bifurcation boundary's side, on which the parameters are located. If parameter $a_{2}$ is moved to 3 with the other parameters set as in the appendix table, the solution becomes periodic. See the solution time path in Figure 2 for output gap.

Period-doubling bifurcation can arise when the central banker reacts aggressively to the expected future values of the output gap. Along the bifurcation boundary in the Figure 1, the values of parameter $a_{2}$ are within the range of 2.75 and 3. At those settings, the central banker actively reacts to the expected future values of inflation and even more aggressively to the forecast based values of the output gap in the forward-looking Taylor rule. 
Figure 1.

Period Doubling Bifurcation Boundary for a New Keynesian Model with a ForwardLooking Taylor Rule

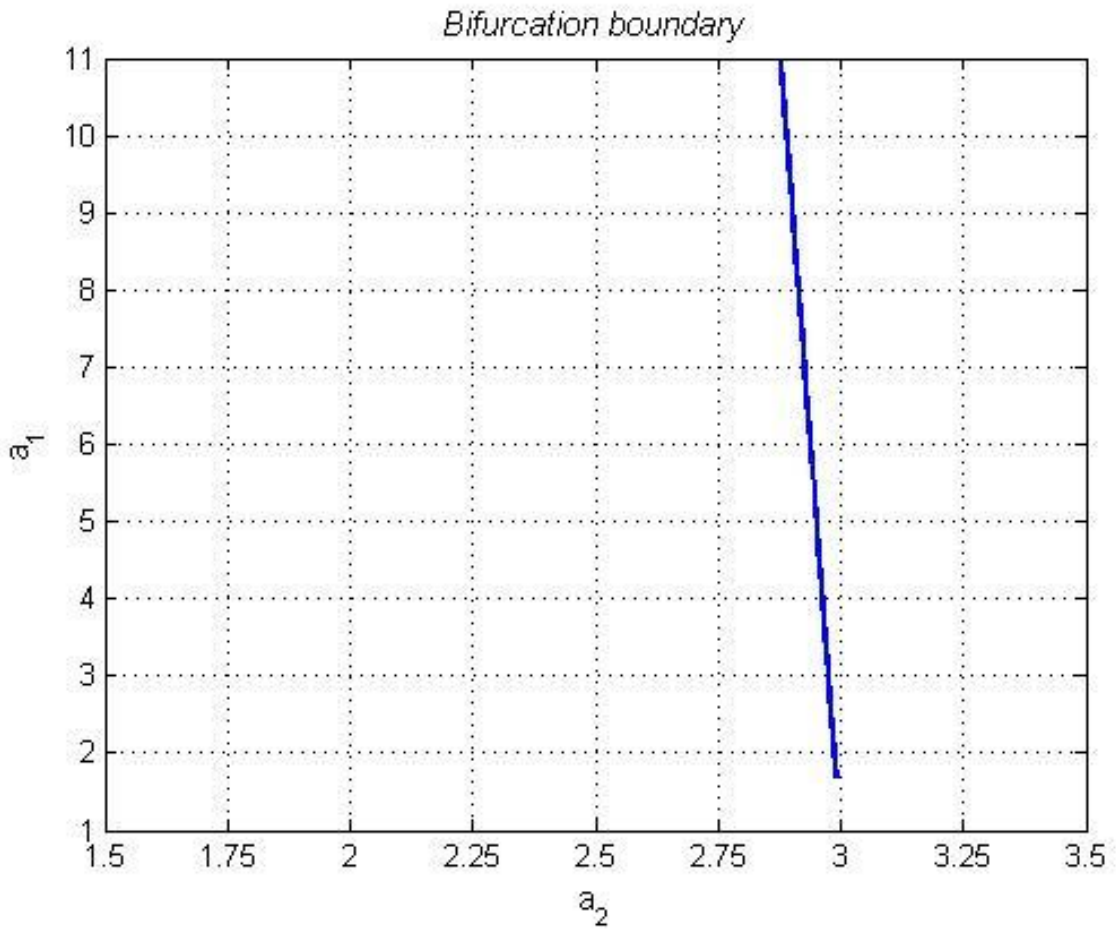

Figure 2.

Fixed Point Curve for a New Keynesian Model with Forward-Looking Taylor Rule

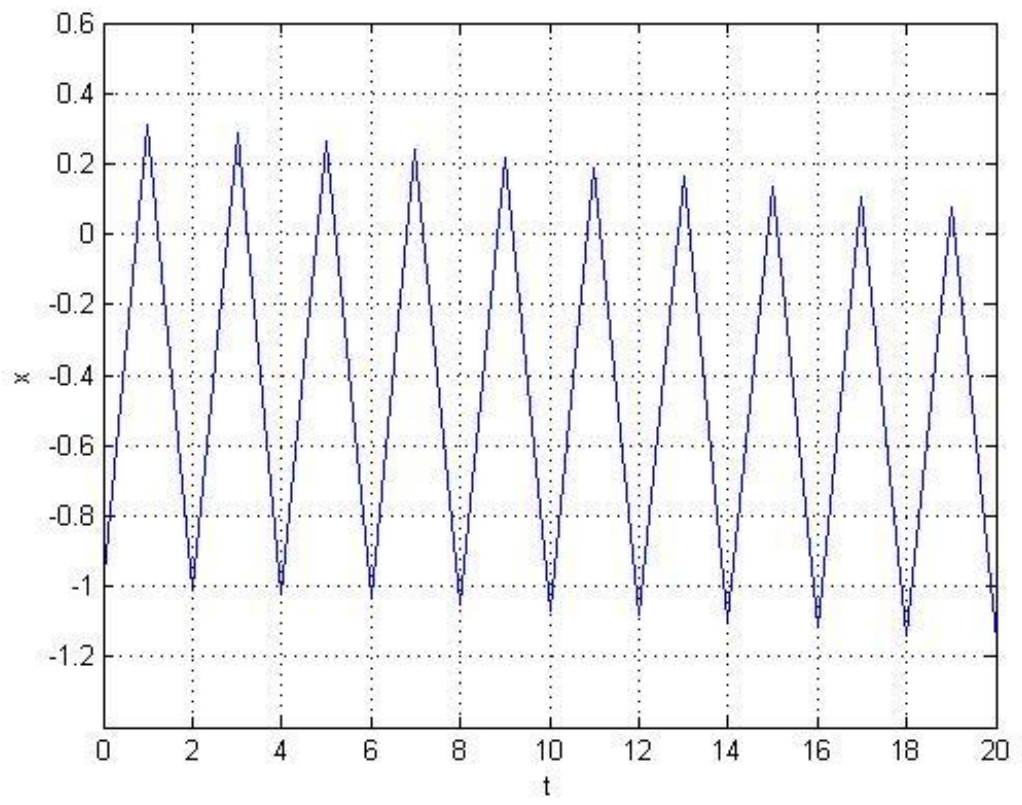




\subsection{Hybrid Taylor Rule:}

Consider the Taylor rule of the following form:

$$
i_{t}=a_{1} E_{t} \pi_{t+1}+a_{2} x_{t}
$$

where the interest rate is set according to forward-looking inflation and currentlooking output gap. A rule of that form was proposed in Clarida, Gali and Gertler (2000) and is purported to capture the central bank's existing policy.

Candidates for bifurcation parameters are the coefficients of the monetary policy rule, $a_{1}$ and $a_{2}$. We shall need the following proposition, proved in Barnett and Duzhak (2008).

Proposition 3.3: The new Keynesian model with Hybrid-Taylor rule, equations (2.1), (2.2), (3.3), undergoes a Hopf bifurcation, if and only if the discriminant of the characteristic polynomial is negative and $a_{2}^{c}=\beta \sigma-\sigma$.

We can combine the critical value for $a_{2}$ with the condition on the discriminant of the characteristic polynomial to provide the condition defining the Hopf bifurcation boundary. The resulting bifurcation boundary is the set of parameter values satisfying the following condition:

$$
-1<\frac{\sigma\left(1+\beta^{2}\right)-\kappa\left(a_{1}-1\right)}{2 \sigma \beta}<1 .
$$

\subsection{Current-Looking Inflation Targeting}

We now use the inflation targeting equation

$$
i_{t}=a_{1} \pi_{t},
$$

instead of the Taylor rule, as the third equation for New Keynesian model.

The model is parameterized by: 


$$
\boldsymbol{\varphi}=\left(\begin{array}{c}
\beta \\
\sigma \\
\kappa \\
a_{1}
\end{array}\right) .
$$

We need to choose a bifurcation parameter to vary, while holding other parameters constant. A candidate for a bifurcation parameter is the coefficient, $a_{1}$, of the monetary policy rule. We have the following proposition about the current-looking inflation targeting New Keynesian model. The proof is in Barnett and Duzhak (2008).

Proposition 3.4: The New Keynesian model with current-looking inflation targeting, equations, (2.1), (2.2), and (3.4), produces a Hopf bifurcation, if and only if the

discriminant of the characteristic equation is negative and $a_{1}^{c}=\frac{\sigma \beta-\sigma}{\kappa}$.

We can combine the critical value for $a_{1}$ with the condition on the discriminant of the characteristic polynomial to provide the condition defining the Hopf bifurcation boundary. The resulting bifurcation boundary is the set of parameter values satisfying the following condition:

$$
-3<\frac{\sigma+\kappa}{\sigma \beta}<1
$$

\subsection{Forward-Looking Inflation Target Rule}

Now we use the following forward-looking inflation targeting rule,

$$
i_{t}=a_{1} E_{t} \pi_{t+1},
$$

instead of the current-looking rule, as the third equation for the New Keynesian model.

We have the following proposition about the forward-looking inflationtargeting New Keynesian model. Surprisingly this result does not require separate 
setting of $a_{1}$ to attain Hopf bifurcation. Under the conditions of this proposition, no freedom remains to select $a_{1}$ independently. The proof of the proposition is in Barnett and Duzhak (2008).

Proposition 3.5: The New Keynesian model, (2.1), (2.2), (3.5), with forward-looking inflation targeting produces a Hopf bifurcation, if and only if the discriminant of the characteristic equation (3.9) is negative and $\beta^{c}=1$.

We can combine the critical value for $\beta$ with the condition on the discriminant of the characteristic polynomial to provide the condition defining the Hopf bifurcation boundary. The resulting bifurcation boundary is the set of parameter values satisfying the following condition:

$$
-3<\frac{\kappa\left(a_{1}-1\right)}{2 \sigma}<1 .
$$

Parameter $\beta$ is the discount factor from the firm's optimization problem and is also a coefficient in the Phillips curve scaling the impact of expected inflation. Some authors assume for simplicity that $\beta=1 .^{7}$ Surprisingly we find that that setting can put the New Keynesian model with forward-looking inflation targeting directly on top of a Hopf bifurcation boundary, and hence can induce instability. This conclusion is conditional upon the assumption that the log-linearized New Keynesian model is a good approximation to the economy and that the discriminant of the characteristic equation is negative. In such cases, setting the discount factor $\beta$ equal to unity is not appropriate.

If the model is parameterized by discount factor $\beta=0.98$, then the dynamic

\footnotetext{
${ }^{7}$ See, e.g., Roberts (1995) and Gali and Gertler (1999).
} 
solution in phase space (inflation rate plotted against output gap) will be periodic, as is demonstrated on Figure 3. It is interesting to see what happens to that solution path, if the parameter value is located directly on the bifurcation boundary. In that case, the solution in phase space will become an invariant limit cycle, as shown in Figure 4.

Figure 3.

Phase Space Solution Path for a New Keynesian Model with Forward-Looking Inflation Target.

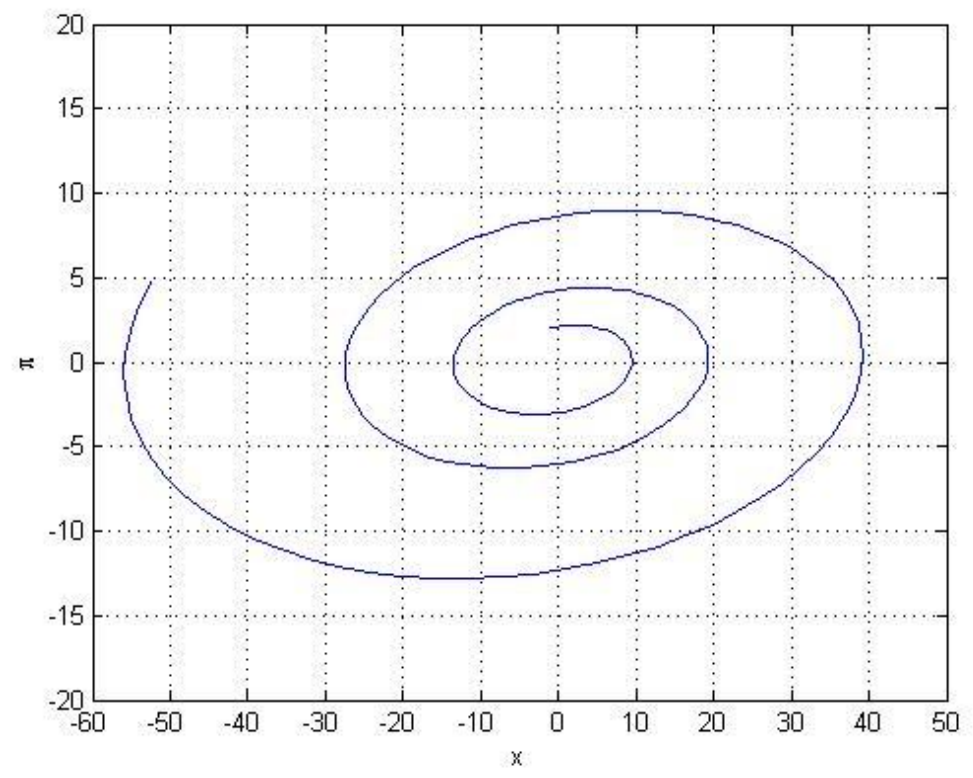




\section{Figure 4.}

Phase Space Solution Path for a New Keynesian Model with Forward-Looking Inflation Target.

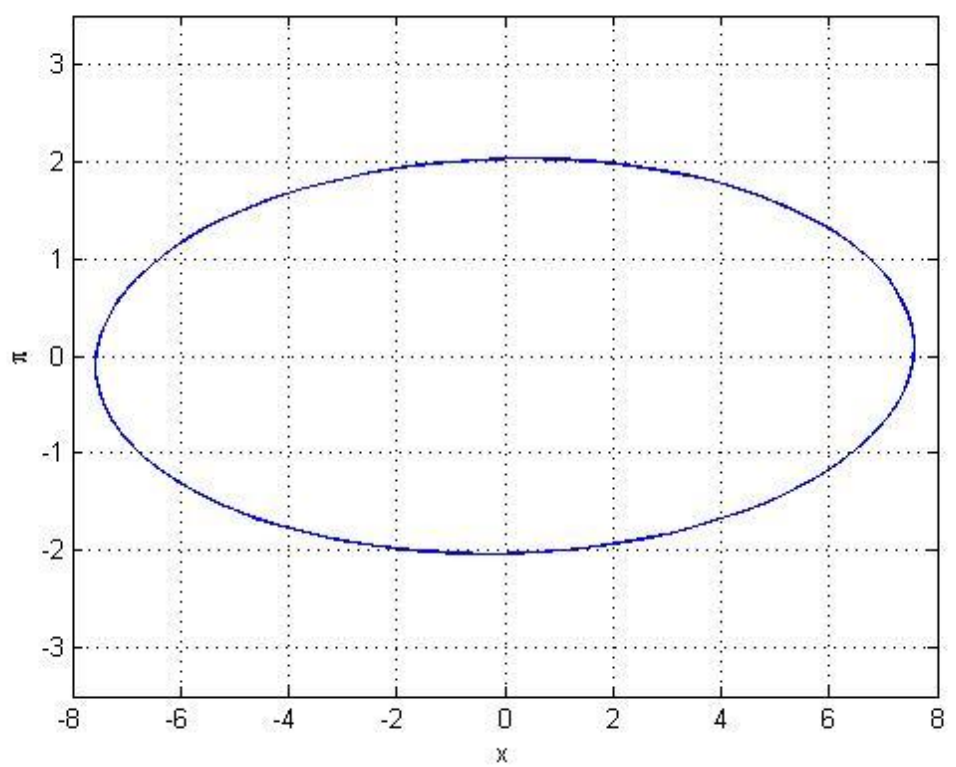

\subsection{Backward Looking Taylor Rule}

Backward-looking rules are constructed in such a way that the monetary authority is making its decisions based on observed past values of the variables. Backward-looking monetary policy rules are intended to prevent expectations driven fluctuations. See, for example, Carlstrom and Fuerst (2000), who argue for a backward-looking interest rate rule reacting aggressively to past values of inflation. Such a policy should be sufficient for determinacy of equilibria. Similar results can be found in Eusepi (2005).

With a backward-looking Taylor rule, the central bank sets an interest rate according to the past values of inflation and output gap as follows:

$$
i_{t}=a_{1} \pi_{t-1}+a_{2} x_{t-1} \text {. }
$$

The New Keynesian model with backward-looking Taylor rule produces the following system: 


$$
\begin{aligned}
& E_{t} \mathbf{x}_{t+1}=\mathbf{C} \mathbf{x}_{t} \text {, where } \\
& \mathbf{x}_{\mathbf{t}}=\left[\begin{array}{c}
x_{t} \\
\pi_{t} \\
i_{t}
\end{array}\right] \text {, and } \mathbf{C}=\left[\begin{array}{ccc}
1+\frac{\kappa}{\sigma \beta} & -\frac{1}{\sigma \beta} & \frac{1}{\sigma} \\
-\frac{\kappa}{\beta} & \frac{1}{\beta} & 0 \\
a_{2} & a_{1} & 0
\end{array}\right] .
\end{aligned}
$$

Matrix $\mathrm{C}$ has the characteristic polynomial $P(\lambda)$

$$
P(\lambda)=\lambda^{3}+z \lambda^{2}+y \lambda+x
$$

where

$$
\begin{aligned}
& z=-\frac{\sigma(1+\beta)+\kappa}{\sigma \beta}, \\
& y=\frac{\sigma-\beta a_{2}}{\sigma \beta}, \\
& x=\frac{\kappa a_{1}+a_{2}}{\sigma \beta} .
\end{aligned}
$$

For the New Keynesian model with backward-looking monetary policy rule, we need the following proposition.

Proposition 3.6: The New Keynesian model with backward-looking Taylor rule produces a Hopf bifurcation, if the transversality condition, $\left.\frac{\partial\left|\lambda_{j}\left(\mathbf{x}^{*}, \boldsymbol{\varphi}^{*}\right)\right|}{\partial \varphi_{i}^{*}}\right|_{\varphi_{i}^{*}=\varphi_{i}^{c}} \neq 0$, holds, and if the parameters $\alpha_{1}$ and $\alpha_{2}$ satisfy the following three conditions at the equilibrium:
(a) $\left|\frac{a_{2}+\kappa a_{1}}{\sigma \beta}\right|<1$,
(b) $a_{2}(1-\beta)+\kappa\left(a_{1}-1\right)>0$,
(c) $\frac{\sigma-\beta a_{2}}{\sigma \beta}+\frac{\left(\kappa a_{1}+a_{2}\right)(\sigma(1+\beta)+\kappa)}{\sigma^{2} \beta^{2}}=1-\left(\frac{\kappa a_{1}+a_{2}}{\sigma \beta}\right)^{2}$. 
Proof: After we apply Theorem 3.2 to the characteristic polynomial, (3.7), we acquire conditions (a), (b) and (c) below, corresponding to the three conditions necessary for a Hopf bifurcation in Theorem 3.

(a) $\left|\frac{a_{2}+\kappa a_{1}}{\sigma \beta}\right|<1$, or equivalently

(a.1) $a_{2}+\kappa a_{1}-\sigma \beta<0$,

(a.2) $a_{2}+\kappa a_{1}+\sigma \beta>0$.

(b) $\left|\frac{a_{2}+\kappa a_{1}-\sigma(1+\beta)-k}{\sigma \beta}\right|<\frac{\sigma \beta+\sigma-\beta a_{2}}{\sigma \beta}$, or equivalently

(b.1) $\left(a_{2}-2 \sigma\right)(1+\beta)+\kappa\left(a_{1}-1\right)<0$,

(c) $\frac{\sigma-\beta a_{2}}{\sigma \beta}+\frac{\left(\kappa a_{1}+a_{2}\right)(\sigma(1+\beta)+\kappa)}{\sigma^{2} \beta^{2}}=1-\left(\frac{\kappa a_{1}+a_{2}}{\sigma \beta}\right)^{2}$.

Note, that the modulus condition (b) is equivalent to only one algebraic condition, unlike modulus condition (a), which is equivalent to the two conditions, (a.1) and (a.2). The reason is that the expression inside the modulus in (b) cannot be negative, as can be seen as follows. We can re-write the expression inside the modulus of (b) as

$$
\frac{a_{2}+\kappa a_{1}-\sigma \beta-(\kappa+\sigma)}{\sigma \beta} .
$$

We know from condition (a.1) that $a_{2}+\kappa a_{1}-\sigma \beta<0$. Also parameters $\beta, \kappa$ and $\sigma$ are positive. Therefore, it follows that

$$
\frac{a_{2}+\kappa a_{1}-\sigma \beta-(\kappa+\sigma)}{\sigma \beta}<0,
$$

so the sign of the expression inside the modulus in (b) must be positive.

We have proved that conditions (a)-(c) are of the correct form. Therefore, according to Theorem 3.2, the system consisting of equations (2.1), (2.2), and (3.6) will have a pair of complex conjugate eigenvalues on the unit circle and one eigenvalue inside the unit circle. 
Let $\varphi_{i}^{*}$ be a bifurcation parameter such that at the critical value $\varphi_{i}^{c}$ a pair of complex conjugate eigenvalues reach the unit circle and the other eigenvalue is located inside the unit circle. According to Theorem 3.2, parameter $\varphi_{i}^{*}$ satisfies conditions (a) -(c). Once the bifurcation parameter has been chosen and $\varphi_{i}^{c}$ located, a Hopf bifurcation will occur, if the transversality condition $\left.\frac{\partial\left|\lambda_{j}\left(\mathbf{x}^{*}, \varphi^{*}\right)\right|}{\partial \varphi_{i}^{*}}\right|_{\varphi_{i}^{*}=\varphi_{i}^{c}} \neq 0$ also holds. ${ }^{8}$

\subsection{Backward looking inflation targeting.}

Backward looking inflation targeting sets the interest rate according to past values of inflation, as follows:

$$
i_{t}=a_{1} \pi_{t-1} .
$$

New Keynesian models consisting of equations (2.1), (2.2), (3.8) have the following Jacobian:

$$
\mathrm{J}=\left[\begin{array}{ccc}
1+\frac{\kappa}{\sigma \beta} & -\frac{1}{\sigma \beta} & \frac{1}{\sigma} \\
-\frac{\kappa}{\beta} & \frac{1}{\beta} & 0 \\
0 & a_{1} & 0
\end{array}\right] .
$$

Jacobian J has the characteristic polynomial

$$
P(\lambda)=\lambda^{3}+z \lambda^{2}+y \lambda+x,
$$

where

$$
\begin{aligned}
& z=-\frac{\sigma(1+\beta)+\kappa}{\sigma \beta}, \\
& y=\frac{1}{\beta}, \\
& x=\frac{\kappa a_{1}}{\sigma \beta} .
\end{aligned}
$$

${ }^{8}$ See Wen, Xu, and Han (2002). 
Proposition 3.7: The New Keynesian model with backward-looking inflation targeting produces a Hopf bifurcation, if parameters $\varphi_{i}^{*}$ satisfy conditions (a)-(c) and if the transversality condition $\left.\frac{\partial\left|\lambda_{j}\left(\mathbf{x}^{*}, \varphi^{*}\right)\right|}{\partial \varphi_{i}^{*}}\right|_{\varphi_{i}^{*}=\varphi_{i}^{c}} \neq 0$ holds, where conditions (a)(c) are
(a) $\left|\frac{\kappa a_{1}}{\sigma \beta}\right|<1$
(b) $a_{1}<1$,
(c) $\frac{\sigma^{2} \beta+\kappa a_{1}(\sigma(1+\beta)+\kappa)}{\sigma^{2} \beta^{2}}=1-\left(\frac{\kappa a_{1}}{\sigma \beta}\right)^{2}$.

Proof:

We apply Theorem 3.2 to the Jacobian, J. Conditions (a), (b), and (c) below correspond to the three conditions that are necessary for a Hopf bifurcation in Theorem 3.2:

(a) $\left|\frac{\kappa a_{1}}{\sigma \beta}\right|<1$, or equivalently

(a.1) $\kappa a_{1}-\sigma \beta<0$,

(a.2) $\kappa a_{1}+\sigma \beta>0$,

(b) $\left|\frac{\kappa\left(a_{1}-1\right)-\sigma(1+\beta)}{\sigma \beta}\right|<\frac{\beta+1}{\beta}$, or equivalently

(b.1) $\kappa\left(a_{1}-1\right)-2 \sigma(1+\beta)<0$,

(b.2) $\kappa\left(a_{1}-1\right)>0$,

(c) $\frac{\sigma^{2} \beta+\kappa a_{1}(\sigma(1+\beta)+\kappa)}{\sigma^{2} \beta^{2}}=1-\left(\frac{\kappa a_{1}}{\sigma \beta}\right)^{2}$. 
Condition (b.1) is redundant again, as in the proof of Proposition 3.6. Assuming that condition (a.1) holds, we can re-write (2.1) as:

$$
\kappa a_{1}-\kappa-2 \sigma-2 \sigma \beta<0 .
$$

Rearranging the coefficients, gives the following:

$$
\left(\kappa a_{1}-\sigma \beta\right)-\kappa-2 \sigma-\sigma \beta<0 .
$$

Thus, assuming that (a.1) holds and that all of the coefficients are nonnegative, condition (b.1) will hold. Therefore, we do not need to consider that condition separately, and we can limit our attention to condition (b.2).

Condition (a.2) will always hold, because of the nonnegativity of the coefficients. Condition (b.2) is equivalent to $a_{1}>1$. Consequently, we have all of the conditions necessary from Proposition 3.7.

Hence, for some $\varphi_{i}^{*}$ satisfying conditions (a)-(c), a pair of complex conjugate eigenvalues reaches the unit circle and the other eigenvalue is located inside the unit circle. Once the free bifurcation parameter has been chosen and $\varphi_{i}^{c}$ located, Hopf bifurcation will occur, if the transversality condition $\left.\frac{\partial\left|\lambda_{j}\left(\mathbf{x}^{*}, \varphi^{*}\right)\right|}{\partial \varphi_{i}^{*}}\right|_{\varphi_{i}^{*}=\varphi_{i}^{c}} \neq 0$ also is satisfied.

By this theorem, we have proven theoretically that Hopf bifurcation can occur in the New Keynesian model with a backwards-looking policy rule. Our numerical search for other kinds of bifurcation in this class of models has not found any kinds of bifurcation other than Hopf.

\subsection{Current-Looking Taylor Rule with Interest Rate Smoothing Term}

If the interest rate path is computed from the optimal interest rate rule, it will be much more volatile than the one observed in practice. Central bankers prefer to avoid volatility in interest rates. To smooth interest rates in models, some economists include a lagged interest rate term in the interest rate rule, as follows:

$$
i_{t}=\left(1-a_{3}\right)\left(a_{1} \pi_{t}+a_{2} x_{t}\right)+a_{3} i_{t-1} .
$$


This type of rule produces a Taylor rule with interest rate smoothing. In this case, we have the following set of parameters:

$$
\boldsymbol{\varphi}=\left(\begin{array}{c}
\beta \\
\sigma \\
\kappa \\
a_{1} \\
a_{2} \\
a_{3}
\end{array}\right) .
$$

Parameter $a_{3}$ describes the degree of interest rate smoothing by the central bank and is assumed to be between zero and one.

The new Keynesian model with current-looking Taylor rule and interest rate smoothing has the following matrix form:

$E_{t} \mathbf{x}_{t+1}=\mathbf{C} \mathbf{x}_{t}$, where

$$
\begin{aligned}
& \mathbf{x}_{\mathbf{t}}=\left[\begin{array}{c}
x_{t} \\
\pi_{t} \\
i_{t}
\end{array}\right], \\
& \mathbf{C}=\left[\begin{array}{ccc}
1+\frac{k}{\sigma \beta} & -\frac{1}{\sigma \beta} & \frac{1}{\sigma} \\
-\frac{\kappa}{\beta} & \frac{1}{\beta} & 0 \\
-a_{2}\left(a_{3}-1\right)+\frac{\left(-1+a_{3}\right)\left(a_{1} \sigma-a_{2}\right) \kappa}{\sigma \beta} & -\frac{\left(-1+a_{3}\right)\left(a_{1} \sigma-a_{2}\right)}{\sigma \beta} & -\frac{a_{2}\left(-1+a_{3}\right)}{\sigma}+a_{3}
\end{array}\right] .
\end{aligned}
$$

This system produces the following characteristic polynomial:

$$
P(\lambda)=\lambda^{3}+z \lambda^{2}+y \lambda+x
$$

where

$$
\begin{aligned}
& x=-\frac{a_{3}}{\beta}, \\
& y=\frac{\kappa a_{1}-a_{2} a_{3}+a_{2}+\kappa a_{3}\left(1-a_{1}\right)}{\sigma \beta}+a_{3}+\frac{1+a_{3}}{\beta}, \\
& z=\frac{a_{2}\left(a_{3}-1\right)}{\sigma}-1-a_{3}-\frac{\kappa}{\sigma \beta}-\frac{1}{\beta} .
\end{aligned}
$$

Proposition 3.8: The New Keynesian model consisting of equations (2.1), (2.2), (3.10) produces a Hopf bifurcation, if parameters $\varphi_{i}^{*}$ satisfy conditions (a)-(c) and if the transversality condition $\left.\frac{\partial\left|\lambda_{j}\left(\mathbf{x}^{*}, \boldsymbol{\varphi}^{*}\right)\right|}{\partial \varphi_{i}^{*}}\right|_{\varphi_{i}^{*}=\varphi_{i}^{c}} \neq 0$ holds, where conditions (a)-(c) are: 
(a) $a_{3}-\beta<0$,

(b) $a_{1}>1$,

(c) $\frac{1-a_{3}^{2}}{\beta}-\left(1-a_{3}\right)+\frac{a_{3}\left(a_{3}-1\right)}{\beta^{2}}+\frac{a_{3} a_{2}\left(a_{3}-2\right)+\kappa a_{1}\left(1-a_{3}\right)+a_{2}+\kappa a_{3}}{\sigma \beta}=0$.

Proof: To find whether Hopf bifurcation is possible in the case of interest rate smoothing, we apply Theorem 3.2. Substituting the coefficients of characteristic polynomial (3.11) into the three conditions of the Theorem 3.2, we get conditions (a)-(c), as follows:

(a) $\left|\frac{a_{3}}{\beta}\right|<1$, or equivalently

(a.1) $a_{3}-\beta<0$,

(a.2) $a_{3}+\beta>0$,

(b) $\left|\frac{a_{2}\left(a_{3}-1\right)}{\sigma}-1-a_{3}-\frac{\kappa}{\sigma \beta}-\frac{1}{\beta}-\frac{a_{3}}{\beta}\right|<1+a_{3}+\frac{\kappa a_{1}-a_{2} a_{3}+a_{2}+\kappa a_{3}\left(1-a_{1}\right)}{\sigma \beta}+\frac{1+a_{3}}{\beta}$,

or equivalently

$$
\begin{gathered}
\text { (b.1) }-\left[\frac{\kappa a_{1}\left(1-a_{3}\right)+\kappa\left(1+a_{3}\right)+a_{2}\left(1-a_{3}\right)}{\sigma \beta}\right]-2\left(1+a_{3}\right)-\frac{a_{2}\left(1-a_{3}\right)}{\sigma}-\frac{2}{\beta}<0, \\
\text { (b.2) } \frac{\kappa\left(a_{1}-1\right)\left(1-a_{3}\right)+a_{2}\left(1-a_{3}\right)(1-\beta)}{\sigma \beta}>0, \\
\text { (c) } \frac{\kappa a_{1}-a_{2} a_{3}+a_{2}+\kappa a_{3}\left(1-a_{1}\right)}{\sigma \beta}+a_{3}+\frac{1+a_{3}}{\beta}-\left(\frac{a_{2} \beta\left(a_{3}-1\right)-\kappa+\sigma-\sigma \beta\left(1+a_{3}\right)}{\sigma \beta}\right) \frac{a_{3}}{\beta}=1-\frac{a_{3}^{2}}{\beta^{2}} .
\end{gathered}
$$

Given that parameters $a_{3}$ and $\beta$ are nonnegative, condition (a.2) would always hold. That leaves us with condition (a.1), which would hold whenever the interest smoothing parameter $a_{3}$ exceeds the discount parameter $\beta$.

Condition (b.1) is always negative, since all of its parameters must be nonnegative. Condition (b.2) would stay positive, if $a_{1}>1$, as long as $a_{3} \neq 1$, since values for $a_{3}$ and $\beta$ are between zero and one.

Algebraic simplification yields the desired condition (c) of Proposition 3.8. Assuming that the transversality condition holds, it follows from Theorem 3.2 that parameters satisfying conditions (a)-(c) will produce a Hopf bifurcation. 


\subsection{Backward-Looking Taylor Rule with Interest Rate Smoothing}

Backward-looking rules are commonly viewed to be the least prone to indeterminacy, and thereby commonly advocated for use by monetary authorities. We have considered backward-looking interest rate rules and inflation targeting. Now, we introduce an interest rate smoothing term. The backward-looking Taylor rule with interest rate smoothing is specified as follows:

$$
i_{t}=\left(1-a_{3}\right)\left(a_{1} \pi_{t-1}+a_{2} x_{t-1}\right)+a_{3} i_{t-1} .
$$

Incorporating equation (3.12) into the New Keynesian model produces the following Jacobian, J:

$$
\mathbf{J}=\left[\begin{array}{ccc}
1+\frac{k}{\sigma \beta} & -\frac{1}{\sigma \beta} & \frac{1}{\sigma} \\
-\frac{\kappa}{\beta} & \frac{1}{\beta} & 0 \\
a_{2}\left(1-a_{3}\right) & a_{1}\left(1-a_{3}\right) & a_{3}
\end{array}\right] .
$$

Jacobian J has characteristic polynomial

$$
P(\lambda)=\lambda^{3}+z \lambda^{2}+y \lambda+x,
$$

where

$$
\begin{aligned}
& x=\frac{\kappa a_{1}\left(1-a_{3}\right)+a_{2}\left(1-a_{3}\right)-\sigma a_{3}}{\sigma \beta}, \\
& y=\frac{a_{2} \beta\left(a_{3}-1\right)+\kappa a_{3}+\sigma\left(1+a_{3}\right)}{\sigma \beta}+a_{3}, \\
& z=-\left(1+a_{3}+\frac{\kappa+\sigma}{\sigma \beta}\right) .
\end{aligned}
$$

Proposition 3.9: The New Keynesian model consisting of equations (2.1), (2.2), (3.12) produces a Hopf bifurcation, if parameters $\varphi_{i}^{*}$ satisfy conditions (a)-(c) and if the transversality condition $\left.\frac{\partial\left|\lambda_{j}\left(\mathbf{x}^{*}, \boldsymbol{\varphi}^{*}\right)\right|}{\partial \varphi_{i}^{*}}\right|_{\varphi_{i}^{*}=\varphi_{i}^{c}} \neq 0$ holds, where conditions (a)-(c) are
(a) $\left|\frac{\kappa a_{1}\left(1-a_{3}\right)+a_{2}\left(1-a_{3}\right)-\sigma a_{3}}{\sigma \beta}\right|<1$,
(b) $\left|\frac{\kappa a_{1}\left(1-a_{3}\right)+a_{2}\left(1-a_{3}\right)-\sigma a_{3}-\kappa-\sigma}{\sigma \beta}-1-a_{3}\right|<\frac{a_{2} \beta\left(a_{3}-1\right)+\kappa a_{3}+\sigma\left(1+a_{3}\right)}{\sigma \beta}+a_{3}$, 
(c) $\frac{a_{2} \beta\left(a_{3}-1\right)+\kappa a_{3}+\sigma\left(1+a_{3}\right)+\sigma \beta a_{3}}{\sigma \beta}+\frac{\left[\left(a_{2}+\kappa a_{1}\right)\left(1-a_{3}\right)-\sigma a_{3}\right]\left[\sigma \beta\left(1+a_{3}\right)+k+\sigma\right]}{(\sigma \beta)^{2}}=1-$ $\left(\frac{\left[\left(a_{2}+\kappa a_{1}\right)\left(1-a_{3}\right)-\sigma a_{3}\right]}{\sigma \beta}\right)^{2}$.

Proof: In order to prove the existence of a Hopf bifurcation in the dynamic model consisting of equations (2.1), (2.2), and (3.12), we apply Theorem 3.2. Substituting the coefficients of the characteristic polynomial into the three conditions of Theorem 3.2, the resulting conditions (1)-(3) are as follows:

(a) $\left|\frac{\kappa a_{1}\left(1-a_{3}\right)+a_{2}\left(1-a_{3}\right)-\sigma a_{3}}{\sigma \beta}\right|<1$, or equivalently

(a.1) $\left(\kappa a_{1}+a_{2}\right)\left(1-a_{3}\right)-\sigma\left(\beta-a_{3}\right)<0$, (a.2) $\left(\beta-a_{3}\right)>0$,

(b) $\left|\frac{\kappa a_{1}\left(1-a_{3}\right)+a_{2}\left(1-a_{3}\right)-\sigma a_{3}-\kappa-\sigma}{\sigma \beta}-1-a_{3}\right|<\frac{a_{2} \beta\left(a_{3}-1\right)+\kappa a_{3}+\sigma\left(1+a_{3}\right)}{\sigma \beta}+a_{3}$, or equivalently

$$
\begin{aligned}
& \text { (b. } 1)-2\left(1+a_{3}\right)\left[1+\frac{1}{\beta}\right]+\frac{a_{2}\left(1-a_{3}\right)}{\sigma}+\frac{\left(a_{2}+\kappa a_{1}\right)\left(1-a_{3}\right)-\kappa\left(1+a_{3}\right)}{\sigma \beta}<0 \\
& (\text { b. } 2)\left(1-a_{1}\right)>0 \\
& \text { (c) } \frac{a_{2} \beta\left(a_{3}-1\right)+\kappa a_{3}+\sigma\left(1+a_{3}\right)+\sigma \beta a_{3}}{\sigma \beta}+\frac{\left[\left(a_{2}+\kappa a_{1}\right)\left(1-a_{3}\right)-\sigma a_{3}\right]\left[\sigma \beta\left(1+a_{3}\right)+k+\sigma\right]}{(\sigma \beta)^{2}}=1- \\
& \left(\frac{\left[\left(a_{2}+\kappa a_{1}\right)\left(1-a_{3}\right)-\sigma a_{3}\right]}{\sigma \beta}\right)^{2}
\end{aligned}
$$

Algebraic simplification yields the desired conditions (a)-(c) of Proposition 3.9. Condition (a.2) follows, since $\left(\kappa a_{1}+a_{2}\right)\left(1-a_{3}\right)+\sigma\left(\beta-a_{3}\right)$ is positive whenever $\beta>a_{3}$. Condition (b.2) follows from the simplification of $\left(1-a_{3}\right)\left[\frac{a_{2}(1-\beta)+\kappa\left(a_{1}-1\right)}{\sigma \beta}\right]>0$. Given that $a_{3}$ and $\beta$ are between zero and one, the inequality would hold, if $\left(1-a_{1}\right)>0$.

Assuming that the transversality condition holds, it follows from Theorem 3.2 that parameters satisfying conditions (a)-(c) will produce a Hopf bifurcation. 
By numerical procedures, we found the existence of a period-doubling bifurcation in this case. In this procedure, we vary parameter $a_{2}$, while holding other parameters fixed. Under the standard calibration in the appendix, we found the first period doubling bifurcation point at $a_{2}=5.7$. Starting at this point, we then vary $a_{2}$ and $a_{3}$ simultaneously. The resulting period-doubling bifurcation boundary is shown in Figure 7.

\section{Figure 7.}

Period Doubling Bifurcation Boundary for the New Keynesian Model with Interest Smoothed Backward-Looking Taylor Rule

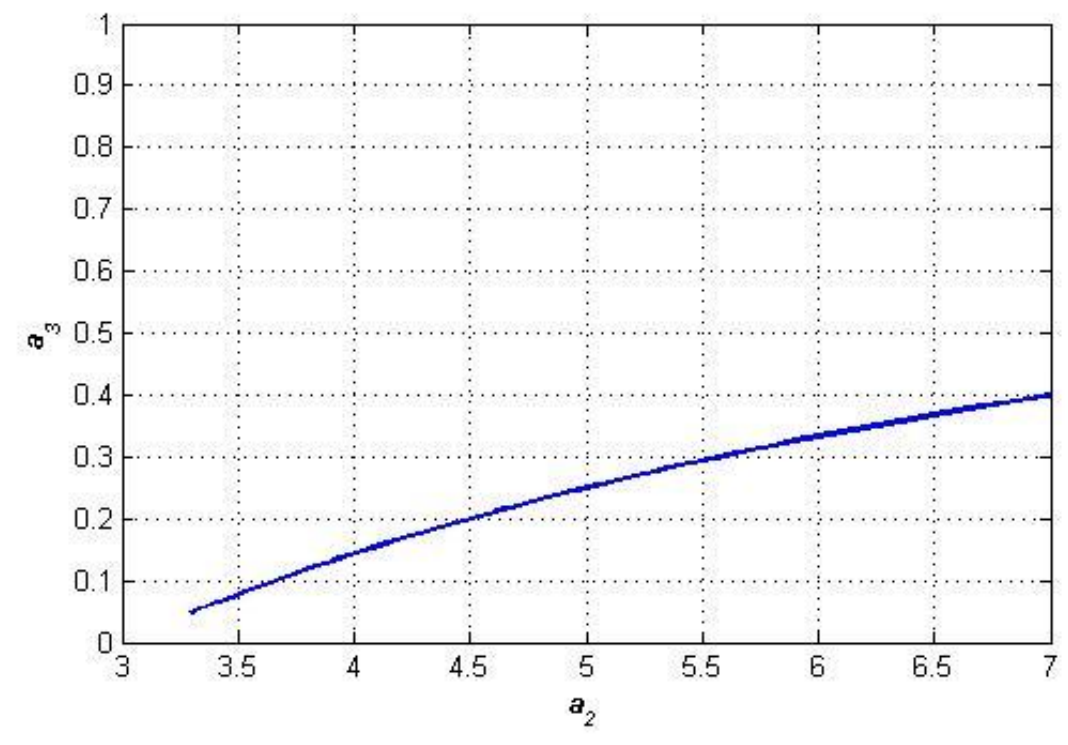

Period doubling bifurcation will occur for large values of the parameter $a_{2}$. In short, aggressive reaction of the central bank to past values of the output gap can lead to a period doubling bifurcation within this model.

Figure 8 displays the section of the period-doubling bifurcation boundary surface acquired by varying parameters $a_{2}$ and $a_{1}$, while holding the other parameters constant. The numerical solution for this boundary was obtained with the starting point $a_{2}=5.7$, and then by simultaneously varying parameters $a_{2}$ and $a_{1}$. 
The located period doubling bifurcation boundary has values of the parameter $a_{2}$ within the range from 5.98 to 6.02 . Hence, a period doubling bifurcation will occur within a very narrow set of parameters $a_{2}$ around 6 . These results were acquired relative to the standard calibration in the appendix. Changing the interest rate smoothing parameter $a_{3}$ leads to a different critical period-doubling bifurcation value for parameter $a_{2}$. See Figure 7. The section of the period-doubling bifurcation surface for parameters $a_{1}$ and $a_{2}$ has similar form, with period-doubling bifurcation appearing within a very narrow set of values for parameter $a_{2}$.

Figure 8.

Period-Doubling Bifurcation Boundary for a New Keynesian Model with an Interest Smoothed Backward-Looking Taylor Rule.

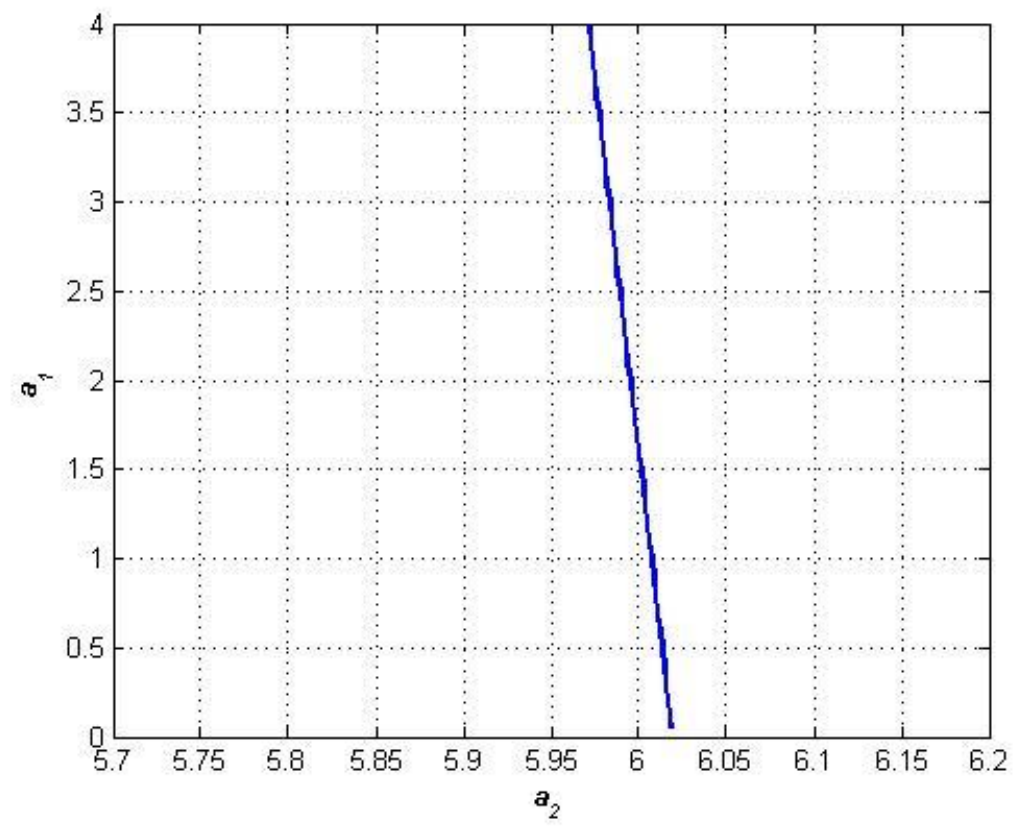

\subsection{Hybrid Rule with Interest Rate Smoothing}

The hybrid rule with interest rate smoothing is proposed in Clarida, Gali and Gertler (1998). This rule specification is widely believed to match the empirics of the monetary policy for the main countries of the European Union, Japan, and the United States. Based on this rule, the central banker sets a short-term interest rate in accordance with a forecast-based value of inflation, the current value of the output gap, and a past value of the interest rate, as follows: 


$$
i_{t}=\left(1-a_{3}\right)\left(a_{1} \pi_{t+1}+a_{2} x_{t}\right)+a_{3} i_{t-1} .
$$

The New Keynesian model consisting of equations (2.1), (2.2), and (3.13) can be represented as follows:

$$
\mathrm{AE}_{\mathrm{t}} \mathbf{X}_{\mathrm{t}+1}=\mathrm{BX}_{\mathrm{t}}
$$

where

$$
\mathbf{A}=\left[\begin{array}{ccc}
1 & \frac{1}{\sigma} & 0 \\
0 & \beta & 0 \\
0 & -a_{1}\left(1-a_{3}\right) & 1
\end{array}\right], \mathbf{B}=\left[\begin{array}{ccc}
1 & 0 & \frac{1}{\sigma} \\
-\kappa & 1 & 0 \\
a_{2}\left(1-a_{3}\right) & 0 & a_{3}
\end{array}\right], \mathbf{x}_{\mathbf{t}}=\left[\begin{array}{c}
x_{t} \\
\pi_{t} \\
i_{t-1}
\end{array}\right] .
$$

This model has the following Jacobian, J:

$$
\mathbf{J}=\left[\begin{array}{ccc}
1+\frac{k}{\sigma \beta} & -\frac{1}{\sigma \beta} & \frac{1}{\sigma} \\
-\frac{\kappa}{\beta} & \frac{1}{\beta} & 0 \\
\frac{a_{1}\left(1-a_{3}\right) \kappa}{\beta}+a_{2}\left(1-a_{3}\right) & -\frac{a_{1}\left(a_{3}-1\right)}{\beta} & a_{3}
\end{array}\right] .
$$

The characteristic polynomial of the Jacobian J is given by:

$$
P(\lambda)=\lambda^{3}+z \lambda^{2}+y \lambda+x,
$$

where

$$
\begin{aligned}
& x=-\frac{a_{3}}{\beta}-\frac{a_{3} a_{2}}{\sigma \beta}+\frac{a_{2}}{\sigma \beta}, \\
& y=a_{3}+\frac{1+a_{3}}{\beta}-\frac{a_{2}\left(1-a_{3}\right)}{\sigma}+\frac{a_{3} \kappa+a_{1} \kappa\left(1-a_{3}\right)}{\sigma \beta}, \\
& z=-\left(1+a_{3}+\frac{1}{\beta}+\frac{\kappa}{\sigma \beta}\right) .
\end{aligned}
$$

Proposition 3.10: The New Keynesian model consisting of equations (2.1), (2.2), (3.13) produces a Hopf bifurcation, if parameters $\varphi_{i}^{*}$ satisfy conditions (a)-(c) and if the transversality condition $\left.\frac{\partial\left|\lambda_{j}\left(\mathbf{x}^{*}, \varphi^{*}\right)\right|}{\partial \varphi_{i}^{*}}\right|_{\varphi_{i}^{*}=\varphi_{i}^{c}} \neq 0$ holds, where conditions (a)-(c) are (a) $\left|-\frac{a_{3}}{\beta}-\frac{a_{3} a_{2}}{\sigma \beta}+\frac{a_{2}}{\sigma \beta}\right|<1$,

(b) $\left|\frac{a_{2}\left(1-a_{3}\right)-\kappa}{\sigma \beta}-1-a_{3}-\frac{1}{\beta}-\frac{a_{3}}{\beta}\right|<1+a_{3}+\frac{1+a_{3}}{\beta}-\frac{a_{2}\left(1-a_{3}\right)}{\sigma}+\frac{a_{3} \kappa+a_{1} \kappa\left(1-a_{3}\right)}{\sigma \beta}$, 
(c) $a_{3}+\frac{1+a_{3}}{\beta}-\frac{a_{2}\left(1-a_{3}\right)}{\sigma}+\frac{a_{3} \kappa+a_{1} \kappa\left(1-a_{3}\right)}{\sigma \beta}+\left(-\frac{a_{3}}{\beta}+\frac{a_{2}\left(1-a_{3}\right)}{\sigma \beta}\right)\left(1+a_{3}+\frac{1}{\beta}+\right.$ $\kappa \sigma \beta=1--a 3 \beta+a 21-a 3 \sigma \beta 2$.

Proof. To prove the possibility of Hopf bifurcation in the system consisting of equations (2.1), (2.2), and (3.13), we apply Theorem 3.2. Substituting the coefficients of the characteristic polynomial, (3.14), into the three conditions of Theorem 3.2, we get conditions (a)-(c) as follows:

(a) $\left|-\frac{a_{3}}{\beta}-\frac{a_{3} a_{2}}{\sigma \beta}+\frac{a_{2}}{\sigma \beta}\right|<1$, or equivalently

$$
\begin{gathered}
\text { (a.1) } a_{2}\left(1-a_{3}\right)-\sigma\left(a_{3}+\beta\right)<0, \\
\text { (a.2) } \beta-a_{3}>0, \\
\text { (b) }\left|\frac{a_{2}\left(1-a_{3}\right)-\kappa}{\sigma \beta}-1-a_{3}-\frac{1}{\beta}-\frac{a_{3}}{\beta}\right|<1+a_{3}+\frac{1+a_{3}}{\beta}-\frac{a_{2}\left(1-a_{3}\right)}{\sigma}+\frac{a_{3} \kappa+a_{1} \kappa\left(1-a_{3}\right)}{\sigma \beta},
\end{gathered}
$$

or equivalently

$$
\begin{aligned}
& \text { (b.1) }-\frac{a_{3}\left(1-a_{2}\right)}{\sigma}-2\left[\frac{1+a_{3}}{\beta}+1+a_{3}\right]+\frac{\left(a_{2}-\kappa a_{1}\right)\left(1-a_{3}\right)-\kappa\left(1+a_{3}\right)}{\sigma \beta}<0, \\
& \text { (b.2) } a_{1}>1,
\end{aligned}
$$

(c) $a_{3}+\frac{1+a_{3}}{\beta}-\frac{a_{2}\left(1-a_{3}\right)}{\sigma}+\frac{a_{3} \kappa+a_{1} \kappa\left(1-a_{3}\right)}{\sigma \beta}+\left(-\frac{a_{3}}{\beta}+\frac{a_{2}\left(1-a_{3}\right)}{\sigma \beta}\right)\left(1+a_{3}+\frac{1}{\beta}+\frac{\kappa}{\sigma \beta}\right)=1-$ $\left(-\frac{a_{3}}{\beta}+\frac{a_{2}\left(1-a_{3}\right)}{\sigma \beta}\right)^{2}$.

Condition (a.2) holds, because parameter $a_{3}$ is nonnegative and less than one. Thus, given the initial inequality $a_{2}\left(1-a_{3}\right)+\sigma\left(\beta-a_{3}\right)>0$, it would be positive whenever $\beta>a_{3}$. Condition (b.2) follows from $\frac{\left(1-a_{3}\right)\left[a_{2}+\kappa\left(a_{1}-1\right)\right]}{\sigma \beta}+\frac{a_{3}\left(1-a_{2}\right)}{\sigma}>0$. We assume that parameters are nonnegative. Furthermore, $a_{3}<1$. Thus the inequality will hold, if $a_{1}>1$. Conditions (a), (b) and (c) are equivalent to those stated in Proposition 3.10. Thus the New Keynesian model consisting of equations (2.1), (2.2), and (3.13) will produce conditions (a)-(c). 
Consequently, for some $\varphi_{i}^{*}$ satisfying conditions (1)-(3), a pair of complex conjugate eigenvalues reach the unit circle, and the other eigenvalue is located inside the unit circle. With the free bifurcation parameter chosen and $\varphi_{i}^{c}$ located, a Hopf bifurcation will occur, if the transversality condition $\left.\frac{\partial\left|\lambda_{j}\left(\mathbf{x}^{*}, \varphi^{*}\right)\right|}{\partial \varphi_{i}^{*}}\right|_{\varphi_{i}^{*}=\varphi_{i}^{c}} \neq 0$ is satisfied.

Numerical analysis of this dynamic system reveals period doubling bifurcation, as follows. We first vary parameter $a_{2}$, while holding other parameters constant. Assuming the standard calibration in the appendix, the critical value of parameter $a_{2}$ is 3.03. Starting with this initial condition, we continue numerically searching for sections of the period doubling bifurcation boundary, first for parameters $a_{2}$ and $a_{3}$ with the other parameters held constant, and then for parameters $a_{2}$ and $a_{1}$ with the other parameters held constant. Bifurcation boundaries for the above cases are illustrated in Figures 9 and Figure 10, respectively. 


\section{Figure 9.}

Period Doubling Bifurcation Boundary for the New Keynesian Model with Hybrid Interest Rate Rule

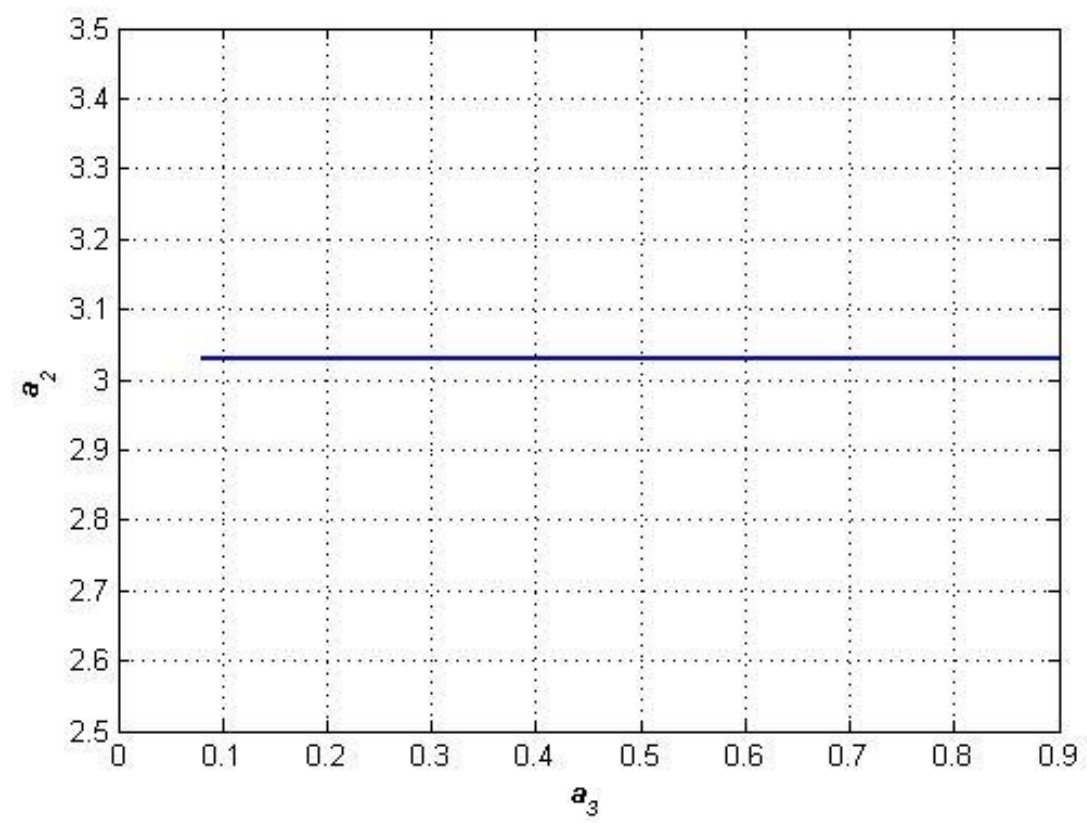

Figure 9 shows the period doubling bifurcation boundary for parameters $a_{2}$ and $a_{3}$. The quality of solution for New Keynesian model with a hybrid type of Taylor rule with interest rate smoothing would change if central banker would start actively reacting to an output gap, with the policy parameter $a_{2}>3$. While simultaneously varying parameters $a_{2}$ and $a_{3}$, we found a fold flip bifurcation point at $a_{2}=3.03$ and $a_{2}=0.46$. At this point one of the eigenvalues equals negative one and another equals one. Once the eigenvalue crosses through this point, the solution becomes highly unstable, since all of the eigenvalues become greater or equal to one in absolute value. 


\section{Figure 10.}

Period Doubling Bifurcation Boundary for the New Keynesian Model with Hybrid Interest Rate Rule

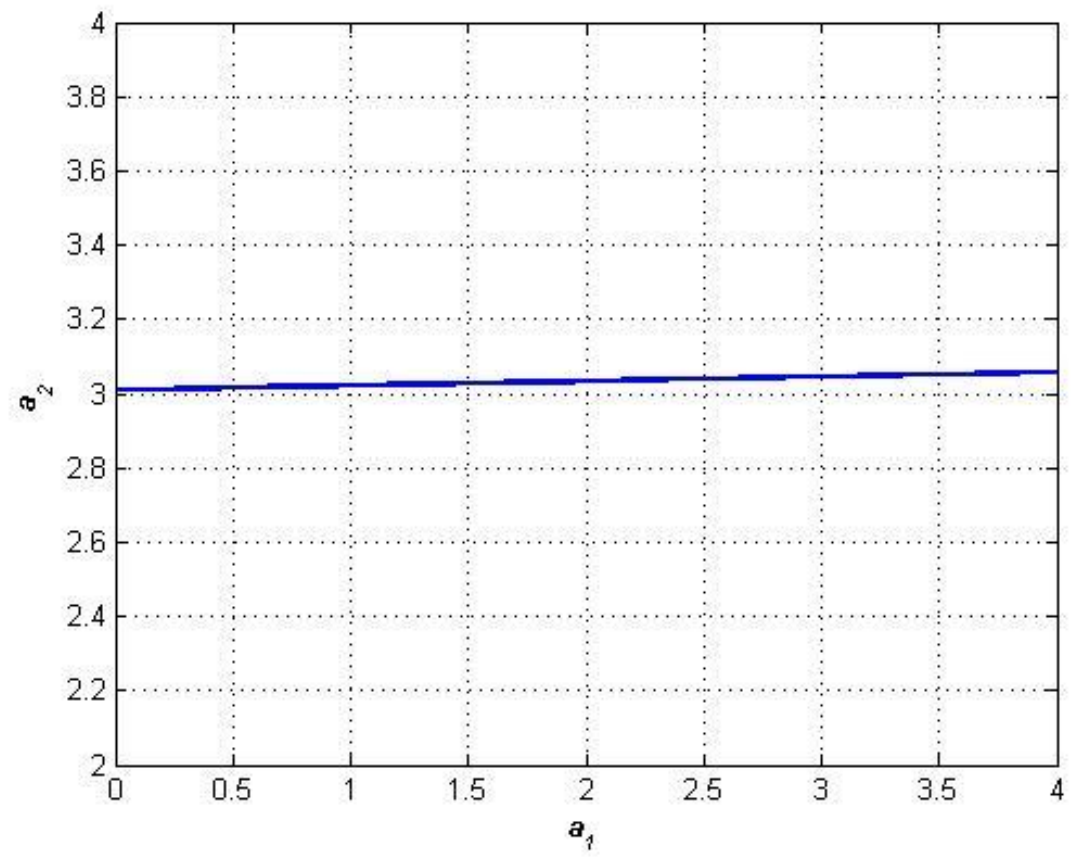

Figure 10 shows the period-doubling bifurcation-boundary section for parameters $a_{2}$ and $a_{1}$, with the other parameters held constant. Within the bifurcation boundary, parameter $a_{2}$ is located mostly between 3 and 3.15, regardless of the values of parameter $a_{1}$. Therefore, given the standard calibration, period doubling bifurcation will occur if the central bank actively reacts to the output gap.

Bifurcation analysis of monetary policy rules with interest rate smoothing reveal two types of bifurcation. The analytical analysis established existence of Hopf bifurcation. The numerical procedure located the possibility of period-doubling bifurcation. Furthermore, period doubling bifurcation was found for several types of policy rules using interest rate smoothing. Even backward-looking interest rate rules show evidence of this type of bifurcation, although previously thought to be the least prone to any kind of bifurcations.

\section{Conclusion}


In this paper, we develop the formulas, prove the propositions, and provide the numerical procedures we are currently using to detect bifurcation boundaries in the parameter spaces of New Keynesian models.

We find two types of bifurcation within the considered New Keynesian functional structures. Our analytical bifurcation analysis detected the possibility of Hopf bifurcation. The numerical bifurcation procedure revealed the possibility of period doubling bifurcation and located that bifurcation boundary. Points along the bifurcation boundaries depend upon the settings of the policy parameters, specifying the central banker's reaction to inflation and output gap. We also introduce a parameter specifying the degree of interest rate smoothing within the corresponding monetary policy rules. The numerical analysis revealed that period doubling bifurcation is most likely to arise in New Keynesian models with the forward-looking monetary policy rules, and in those cases usually occurs for large values of the parameter $a_{2}$, which specifies the central bank's reaction to inflation.

We have been analyzing the reduced log-linearized system. Study of the full nonlinear system will require different tools and will be the subject of future research. In cases in which we did not locate Hopf bifurcation within the theoretically feasible region of the log-linearized system, we cannot conclude that Hopf or other types of bifurcation might not arise in the original nonlinear system. When we find Hopf bifurcation with the linearized system, the result is locally sufficient but not necessary for existence of a bifurcation boundary.

Our future research in this area will also include bifurcation analysis of continuous-time New Keynesian models. There are more tools available for bifurcation analysis of continuous time models than discrete time models. Not only is there more theory, but also there is specialized software for conducting bifurcation analysis with continuous time models. Thus, the results that we expect to acquire will be more precise and complete.

We anticipate that nonlinear analysis and continuous-time analysis will reveal more New Keynesian bifurcation boundaries, not less. 


\section{Appendix}

Table 1. Calibrated parameter values.

\begin{tabular}{|l|l|}
\hline \multicolumn{2}{|l|}{ Structural parameters } \\
\hline$\beta$ & 0.98 \\
\hline$\kappa$ & 0.024 \\
\hline$\sigma$ & 1.5 \\
\hline Policy parameters & \\
\hline$a_{1}$ & 1.5 \\
\hline$a_{2}$ & 0.5 \\
\hline$a_{3}$ & 0.9 \\
\hline
\end{tabular}




\section{References}

Aiyagari, S. R. (1989), “Can there be short-period deterministic cycles when people are long lived?" Quarterly Journal of Economics 104, 163-185.

Andronov, A. A. (1929), "Les Cycles Limits de Poincaré et la Théorie des Oscillations Autoentretenues," Comptes-rendus de l'Academie des Sciences 189, 559-561.

W. A. Barnett and W. A. Duzhak, "Non-robust dynamic inferences from macroeconometric models: Bifurcation stratification of confidence regions," Physica $A$, vol. 387, issue 15 , June 15 , 2008, pp. 3817-3825.

Barnett, William A. and Yijun He (1999), "Stability Analysis of Continuous-Time Macroeconometric Systems," Studies in Nonlinear Dynamics and Econometrics, January, vol 3, no. 4, pp. 169-188.

Barnett, William A. and Yijun He (2001), "Nonlinearity, Chaos, and Bifurcation: A Competition and an Experiment," with Yijun He, in Takashi Negishi, Rama Ramachandran, and Kazuo Mino (eds.), Economic Theory, Dynamics and Markets: Essays in Honor of Ryuzo Sato, Kluwer Academic Publishers, 167-187.

Barnett, William A. and Yijun He (2002), "Stabilization Policy as Bifurcation Selection: Would Stabilization Policy Work if the Economy Really Were Unstable?," Macroeconomic Dynamics, vol 6, no 5, November, 713-747.

Barnett, William A. and Yijun He (2004), "Bifurcations in Macroeconomic Models," in Steve Dowrick, Rohan Pitchford, and Steven Turnovsky (eds), Economic Growth and Macroeconomic Dynamics: Recent Developments in Economic Theory, Cambridge University Press, 95-112.

Barnett, William A. and Yijun He (2006), "Robustness of Inferences to Singularity 
Bifurcation," Proceedings of the Joint Statistical Meetings of the 2005 American Statistical Society, vol. 100, American Statistical Association, February.

Barnett, William A. and Susan He (2008), "Existence of Singularity Bifurcation in an Open-Economy Euler-Equations Model of the United States Economy," Open Economies Review, submitted.

Benhabib, J., Day, R. H. (1982),"A characterization of erratic dynamics in the overlapping generations model." Journal of Economic Dynamics and Control 4, 3755.

Benhabib J., Nishimura K. (1979), The Hopf bifurcation and the existence and stability of closed orbits in multisector models of optimal economic growth, Journal of Economic Theory, vol. 21, pp. 421-444.

Benhabib, J., Rustichini, A. (1991), “Vintage capital, investment and growth.” Journal of Economic Theory 55, 323-339.

Bergstrom, A. R. (1996), "Survey of Continuous Time Econometrics," in W. A. Barnett, G. Gandolfo, and C. Hillinger (eds.), Dynamic Disequilibrium Modeling, Cambridge University Press, 3-26.

Bergstrom, A. R., K. B. Nowmann, and S. Wandasiewicz (1994), "Monetary and Fiscal Policy in a Second-order Continuous Time Macroeconometric Model of the United Kingdom," Journal of Economic Dynamics and Control, 18, 731-761.

Bergstrom, A. R., K. B. Nowman, and C. R. Wymer (1992), "Gaussian Estimation of a Second Order Continuous Time Macroeconometric Model of the United Kingdom," Economic Modelling, 9, 313-352.

Bergstrom, A.R., and C.R. Wymer (1976), "A Model of Disequilibrium Neoclassical 
Growth and its Application to the United Kingdom," in A.R. Bergstrom, ed., Statistical Inference in Continuous Time Economic Models, Amsterdam: North Holland, 267327.

Bergstrom, A. R. and K. B. Nowman (2006), A Continuous Time Econometric Model of the United Kingdom with Stochastic Trends, Cambridge University Press, forthcoming.

Bernanke, Ben S., Thomas Laubach, Frederic S. Mishkin, and Adam S. Posen . (1999), Inflation Targeting: Lessons from the International Experience. Princeton, NJ: Princeton University Press.

Calvo, G. (1983), "Staggered Prices in a Utility-Maximizing Framework," Journal of Monetary Economics, 12, 383-398.

Carlstrom, Charles T. and Timothy S. Fuerst, 2000. "Forward-looking versus backward-looking Taylor rules," Working Paper 0009, Federal Reserve Bank of Cleveland.

Clarida, R., J.Gali and M.Gertler (1998), "Monetary Policy Rules in Practice: Some International Evidence", European Economics Review, June, 1033-1068

Clarida, Richard, Jordi Galí, and Mark Gertler (1999), "The Science of Monetary Policy:

A New Keynesian Perspective," Journal of Economic Literature 37, December, 16611707.

Dixit, Avinash and Joseph E. Stiglitz (1977), "Monopolistic Competition and Optimum Product Diversity," American Economic Review67, 297-308. 
Eusepi, Stefano (2005). "Comparing forecast-based and backward-looking Taylor rules: a "global" analysis," Staff Reports 198, Federal Reserve Bank of New York.

Gali, J., and M. Gertler (1999), "Inflation dynamics: a structural econometric analysis, "Journal of Monetary Economics. 44, October, 195-222.

Gale, D. (1973), "Pure exchange equilibrium of dynamic economic models." Journal of Economic Theory 6, 12-36.

Gandolfo, Giancarlo 1996, Economic Dynamics, Third edition, New York and Heidelburg, Springer.

Gavin, William T (2003), "Inflation Targeting: Why It Works and How To Make It Work Better," Federal Reserve Bank of Saint Louis Working Paper 2003-027B.

Grandmont, J. M. (1985), "On Endogenous Competitive Business Cycles," Econometrica, 53, 995-1045.

Hopf, E. (1942), “Abzweigung Einer Periodischen Lösung von Einer Stationaren Lösung Eines Differetialsystems," Sachsische Akademie der Wissenschaften Mathematische-Physikalische, Leipzig 94, 1-22.

Iooss, G. (1979), Bifurcation of Maps and Applications, Mathematical Studies Vol. 36, North-Holland, Amsterdam.

Kuznetsov,Yu.A (1998). Elements of Applied Bifurcation Theory.Springer - Verlag, New York.

Leeper, E. and C. Sims (1994), “Toward a Modern Macro Model Usable for Policy Analysis," NBER Macroeconomics Annual, pp. 81-117. 
McCallum, B.T. (1999), "Issues in the design of monetary policy rules," Handbook of Macroeconomics, eds. J.B. Taylor and M. Woodford. North-Holland Pub. Co.

Poincaré, H. (1892), Les Methodes Nouvelles de la Mechanique Celeste, GauthierVillars, Paris.

Roberts, J. M. (1995), "New Keynesian Economics and the Phillips Curve", Journal of Money, Credit and Banking, 27(4), November, 975-984.

Seydel, R. (1994), Practical bifurcation and stability analysis, Springer-Verlag, New York.

Shapiro, A. H. (2006), "Estimating the New Keynesian Phillips Curve: A Vertical Production Chain Approach" Federal Reserve Bank of Boston Working Paper No. 0611.

Svensson, Lars E. O. (1999), "Inflation Targeting as a Monetary Policy Rule." Journal of Monetary Economics 43, 607-54.

Taylor, John B. (1999), "A Historical Analysis of Monetary Policy Rules," in John B. Taylor, ed., Monetary Policy Rules, Chicago: University of Chicago Press for NBER, $319-40$.

Torre, V. (1977). Existence of Limit Cycles and Control in Complete Keynesian System by Theory of Bifurcations" Econometrica, Vol. 45, No. 6. (Sept.), pp. 1457 1466.

Walsh, Carl E. (2003), Monetary Theory and Policy, 2nd edition MIT Press: Cambridge Mass. 
Wen, Guilin, Daolin Xu, and Xu Han (2002), "On creation of Hopf bifurcations in discrete-time nonlinear systems," Chaos, Vol. 12, Issue 2, pp. 350-355.

Woodford, Michael (2003), Interest and Prices: Foundations of a Theory of Monetary Policy. Princeton, NJ: Princeton University Press. 